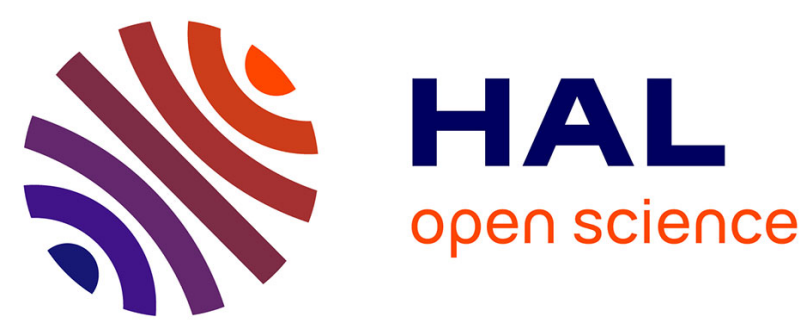

\title{
Linear and nonlinear dynamics of a plate with acoustic black hole, geometric and contact nonlinearity for vibration mitigation
}

\author{
Haiqin Li, Cyril Touzé, François Gautier, Adrien Pelat
}

\section{To cite this version:}

Haiqin Li, Cyril Touzé, François Gautier, Adrien Pelat. Linear and nonlinear dynamics of a plate with acoustic black hole, geometric and contact nonlinearity for vibration mitigation. Journal of Sound and Vibration, 2021, 10.1016/j.jsv.2021.116206 . hal-03222737

\section{HAL Id: hal-03222737 \\ https://hal.science/hal-03222737}

Submitted on 10 May 2021

HAL is a multi-disciplinary open access archive for the deposit and dissemination of scientific research documents, whether they are published or not. The documents may come from teaching and research institutions in France or abroad, or from public or private research centers.
L'archive ouverte pluridisciplinaire HAL, est destinée au dépôt et à la diffusion de documents scientifiques de niveau recherche, publiés ou non, émanant des établissements d'enseignement et de recherche français ou étrangers, des laboratoires publics ou privés. 


\title{
Linear and nonlinear dynamics of a plate with acoustic black hole, geometric and contact nonlinearity for vibration mitigation
}

\author{
Haiqin Li ${ }^{\mathrm{a}, \mathrm{b}, *}$, Cyril Touzéa ${ }^{\mathrm{a}}$, François Gautier ${ }^{\mathrm{b}}$, Adrien Pelat ${ }^{\mathrm{b}}$ \\ ${ }^{a}$ IMSIA, ENSTA Paris-CNRS-EDF-CEA, Institut Polytechnique de Paris, 828 Boulevard des Maréchaux, \\ 91762 Palaiseau Cedex, France \\ ${ }^{b}$ Laboratoire d'Acoustique de l'Université du Mans, UMR CNRS 6613, Avenue Olivier Messiaen, 72085 Le \\ Mans, Cedex 09, France
}

\begin{abstract}
A rectangular plate with a wedge profile creating an Acoustic Black Hole (ABH) termination is studied numerically. A particular emphasis is put on combining two different types of nonlinearity in order to improve the passive damping capacity of the ABH by transferring energy to the high-frequency range where it is more efficient. First, the addition of contact points to create a vibro-impact black hole (VI-ABH) is taken into account, following a previous study on beams. The contact nonlinearity allows for a rapid and efficient transfer of energy. Second, the large-amplitude vibrations of the plate in the ABH region where small thickness is reached, is also considered. The geometric nonlinearity is incorporated using a von Kármán plate model, and the regime of wave turbulence is shown to be triggered thus creating an energy flux from the low to the high frequencies. The linear characteristics of the ABH plate are first analyzed. Numerical results show the appearance of overdamped modes gathered in solution branches with constant number of half-waves in the transverse direction of the $\mathrm{ABH}$, seen as a waveguide. The structure of the branches is shown to be more and more prominent when increasing the width of the plate, showing a transition from beam-like to full plate structure, with a fixed value for the fundamental cut-on frequency. The combination of both contact and geometric nonlinearities to improve the ABH effect is then reported. It is shown that the coexistence of both nonlinearities provides better passive damping efficacy.
\end{abstract}

Keywords: Nonlinear vibration; Acoustic black hole; Energy transfer; Vibro-impact; Wave turbulence

\section{Introduction}

Effective control of unwanted vibrations is a subject of special concern in many fields of engineering. In this realm, there is a growing interest in the passive technique gathered under

\footnotetext{
${ }^{*}$ Corresponding author.

Email address: lihaiqin1992@yahoo.com;Haiqin.Li@univ-lemans.fr (Haiqin Li)
} 
the generic name of Acoustic Black Hole $(\mathrm{ABH})$ effect, see for example this recent review paper [1]. Started from the pioneering work conducted in $[2,3]$, the classical implementation of such an $\mathrm{ABH}$ in a beam or plate structure consists of locally decreasing the thickness according to a power law, and adding the tapered area with a thin viscoelastic damping layer. The former results in an important localization of vibration energy into the tapered termination, while the latter ensures an efficient damping performance in the area to increase the energy losses. In this way, an $\mathrm{ABH}$ can be viewed as an anechoic structural termination localized at the boundary.

An increasing number of investigations have been conducted in the last years to study the behaviour and damping performances of structures embedding $\mathrm{ABH}$. Regarding the application of wave and noise mitigation, a significant reduction of the reflection coefficient of the flexural waves has been demonstrated in many contexts [4-9]. From an engineering point of view, a clear improvement on the modal damping ratios and the modal overlap factors is also evidenced in [10]. In addition, analytical exact solutions for $\mathrm{ABH}$ in beams can be found in more recent contributions $[11,12]$. All these results demonstrates that an ABH is able to localize an incident wave entering the tapered area, and associate this localization of energy to an effective damping property, thus confirming the $\mathrm{ABH}$ as an effective passive vibration mitigation technique. On the other hand, experimental demonstrations on the flexural wave propagating properties in different $\mathrm{ABH}$ structures and their efficiency in vibration damping are also numerous [7, 13-16]. All these studies have confirmed the high efficacy of ABH for vibration damping but also for wave manipulation [17] and energy harvesting [18]. Based on different applications, the optimal designs on the ABH configurations and parameters have also been investigated in depth, see e.g. [19-23] for various structural applications.

Up to now, most of the applications are designed targeting the mid and high frequencies for vibration and noise control. On the other hand, less studies considered the low frequency range and the potential improvements that can be gained in this frequency band. However, it is known in vibroacoustic engineering that most of the needs in terms of vibration reduction relate to low frequencies. In the low frequency range, all experimental characterizations of $\mathrm{ABH}$ structures show that no enhanced damping effect is achieved below a cut-on frequency. An exact definition of this cut-on frequency is still a matter of debate in the literature, and different proposals have been given in the past. A very first definition is based on a geometrical consideration. The cut-on frequency is stated as the frequency below which the wavelength of the incoming wave becomes larger than the characteristic length of the $\mathrm{ABH}$ (typically the length of the wedge or the diameter of the axisymmetric taper) [24, 25]. Another proposal is based on the analysis of the dispersion curves and corresponds to the frequency for which a propagative wave exists only in a localized region in the ABH center, leading to a trapping effect (see [11] for the circular configuration and [26] for the wedge configuration). A third definition is based on the analysis of the $\mathrm{ABH}$ in terms of localized modes [20]. The cut-on frequency is thus the frequency of the first ABH local mode, since the $\mathrm{ABH}$ effect can be interpreted using the concept of critical coupling : The optimal tuning of the $\mathrm{ABH}$ coating layer is obtained when the added damping compensates the leakage effect of the ABH. Although these three definitions describe different physical properties, they are 
all related to the $\mathrm{ABH}$ localisation effect and as a consequence to the $\mathrm{ABH}$ efficiency. In practice, the knowledge of this cut-on frequency is of particular importance for the design of $\mathrm{ABH}$ devices in order to target wave absorption in the desired frequency range.

To overcome the intrisic low-frequency limitation, recent investigations have shown that incorporating a nonlinear phenomenon into the design of an $\mathrm{ABH}$ in order to transfer energy from the low to the high-frequency range, where the damping capacity is more efficient, may be a desirable goal to achieve broadband control. A first study was concerned with the geometric nonlinearity, that in any case should be always taken into account in the modelisation due to the thinness of the profile in the ABH termination. Including the largeamplitude vibrations thanks to a von Kármán plate model, Denis et al. showed that the wave turbulence settling down in plate nonlinear vibration and creating an energy flux through lengthscales, can be effectively excited in order to improve the low-frequency efficacy [27]. Adding an extended platform of constant and small thickness to the $\mathrm{ABH}$ region has also been reported and studied in depth in $[9,28]$, and its beneficial contribution to increase the nonlinear vibrations shown in [27]. Along the same line, a Vibro-impact Acoustic Black Hole (VI-ABH) has been proposed in [29] in order to transfer more efficiently the energy. Indeed, one limitation of the geometric nonlinearity is related to the important timescales needed for the wave turbulence regime to settle down. On the other hand, contact nonlinearity brings about immediate energy transfer, so that very effective improvements have been reported for beam $\mathrm{ABH}$ in [29]. The combination of added linear and nonlinear dampers to an $\mathrm{ABH}$ beam is studied in [30], showing also important improvements toward broadband vibration mitigation. In related fields, other studies considered similar ideas and reported results, see for example the quadratic hysteretic nonlinearity characteristic of micro-inhomogeneous materials investigated in [31] for diminishing the wave speed, or the tuned mass-loaded membranes as vibro-impact attachments on a baseline structure in [32]. Nevertheless, all of these studies focus simply on the 1D beam case with a single type of nonlinearity.

The aim of this paper is to consider the simultaneous effects brought by contact and geometric nonlinearity in order to characterize their combined efficiency for vibration mitigation of an $\mathrm{ABH}$ plate. It is structured as follows. The numerical modeling of the VI-ABH consisting of a von Kármán plate with variable thickness and a local vibro-impact element is formulated in section 2. A linear analysis is then conducted in section 3 in order to assess the modal behaviour of the ABH plate. The numerical results highlight important findings on the organization of the damped eigenfrequencies, with the appearance of overdamped plate modes gathered in solution branches with constant number of half-waves in the ABH region. The cut-on frequency of the system and of each solution branch is discussed and their overall organization studied with respect to the parameters of the system. The effect of vibro-impact and its optimal design is reported in Section 4, and Section 5 reports analyses of the combination of geometric nonlinearity together with vibro-impact. Finally, the concluding remarks are presented in Section 6. 


\section{Modeling of the VI-ABH plate}

This section is devoted to the derivation of the numerical model used to simulate the nonlinear dynamics of a VI-ABH plate including geometric nonlinearity. Most of the details on the numerical model have already been presented in separate contributions. Indeed a geometrically nonlinear von Kármán plate modal has already been derived in [27], while the contact nonlinearity has been presented in [29] in the case of a beam. Both developments are merged here for a complete model, and the presentation is kept at its minimum for a sake of brevity.

\subsection{Von Kármán equation for the nonlinear vibration of plate}

A rectangular plate of length $L$ and width $b$ is shown in Fig. 1. It is in contact with a rigid barrier composed of several contact points as discussed below. The plate is composed of three areas. The first one on the right is a uniform region of constant thickness $h_{0}$. The second is the ABH area of length $l_{A B H}$, where the thickness decreases smoothly from $h_{0}$ to $h_{t}$, along $x$ direction, and following a given power law. The third area is an extension of small thickness added in the left of the plate, which is parameterized by its length $l_{\text {add }}$ that can be tuned in order to increase the nonlinear behaviour of the structure.
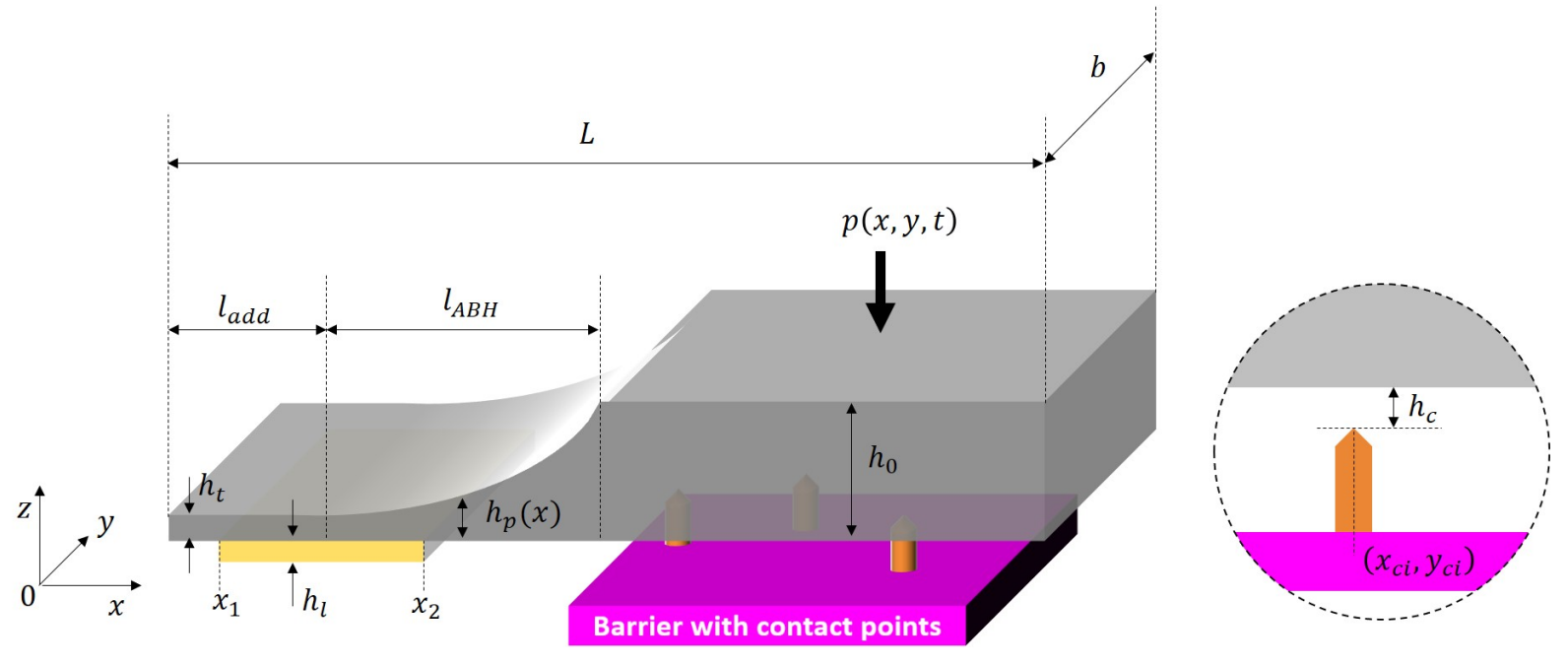

Fig. 1. ABH plate in contact with a rigid barrier

It is emphasized that the thickness of the plate is assumed to vary along the $x$ direction only, hence all the plate characteristics only depends on $x$. According to Fig. 1, the thickness $h_{p}(x)$ of the $\mathrm{ABH}$ plate can be written with respect to the coordinate $x$ as

$$
h_{p}(x)=\left\{\begin{array}{lc}
h_{t} & \forall x \leqslant l_{\text {add }}, \\
h_{t}+\left(h_{0}-h_{t}\right) \frac{\left(x-l_{\text {add }}\right)^{2}}{l_{A B H}^{2}} & \forall x \in\left[l_{\text {add }}, l_{\text {add }}+l_{A B H}\right], \\
h_{0} & \forall x \geqslant l_{\text {add }}+l_{A B H},
\end{array}\right.
$$


Considering the flexural vibration of the plate, taking also into consideration the geometric nonlinearity that could be induced because of the small thickness, the equation of motion for the transverse displacement $w(x, y, t)$ is thus described by the von Kármán [33, 34] plate model with variable thickness, which reads:

$$
\begin{gathered}
\rho(x) h(x) \ddot{w}+\Delta(D(x) \Delta w)-(1-\nu) \mathcal{L}(D(x), w)=p+\mathcal{L}(w, F)+f \\
\Delta\left(\frac{1}{E(x) h(x)} \Delta F\right)-(1+\nu) \mathcal{L}\left(\frac{1}{E(x) h(x)}, F\right)=-\frac{1}{2} \mathcal{L}(w, w)
\end{gathered}
$$

where $D(x)=E(x) h_{p}^{3}(x) / 12\left(1-\nu^{2}\right), E(x), \rho(x), h(x)$ and $\nu$ represent respectively the bending stiffness, Young's modulus, material density, thickness and Poisson ratio of the plate. $p=p(x, y, t)$ is the external force per unit surface, while $f=f(x, y, t)$ refers to the contact force per unit surface. $F=F(x, y, t)$ stands for the Airy stress function. Finally, $\mathcal{L}$ is referred as the Monge-Ampère bilinear operator, whose expression could be found in $[27,33,34]$.

The damping is considered using a Ross-Kerwin-Ungard model by applying modifications on the bending stiffness, density and thickness of the plate which are leading to equivalent or effective mechanical properties. For a viscoelastic layer coated on the plate at the interval $\left[x_{1}, x_{2}\right]$ with thickness $h_{l}$, the complex bending stiffness reads

$$
D^{*}(x)=\left\{\begin{array}{ll}
\frac{E_{p} h_{p}(x)^{3}}{12\left(1-v^{2}\right)}\left(1+j \eta_{p}\right)=D_{p}(x)\left(1+j \eta_{p}\right), & \forall x \notin\left[x_{1}, x_{2}\right] \\
D_{p}(x)\left[\left(1+j \eta_{p}\right)+\frac{E_{l}}{E_{p}}\left(\frac{h_{l}}{h_{p}(x)}\right)^{3}\left(1+j \eta_{l}\right)\right. & \\
3\left(1+\frac{h_{l}}{h_{p}(x)}\right)^{2} \frac{E_{l} h_{l}}{E_{p} h_{p}(x)}\left(1-\eta_{p} \eta_{l}+j\left(\eta_{p}+\eta_{l}\right)\right) \\
+\frac{E_{l} h_{l}}{E_{p} h_{p}(x)}\left(1+j \eta_{l}\right)
\end{array}\right], \forall x \in\left[x_{1}, x_{2}\right]
$$

where $j$ is the imaginary unit, $D_{p}(x), E_{p}, \eta_{p}$ and $h_{p}(x)$ represent respectively the bending stiffness, Young's modulus, loss factor and thickness of the plate without the damping layer, while $E_{l}, \eta_{l}$ and $h_{l}(x)$ are the Young's modulus, loss factor and thickness of the added layer. In this definition, the real bending stiffness $D(x)=\operatorname{Re}\left(D^{*}(x)\right)$ is derived from the real part of $D^{*}(x)$, and the damping is represented by the imaginary part of the complex bending stiffness. In addition, a modification of the thickness $h(x)=h_{b}(x)+h_{l}$ and of the material density $\rho(x)=\left(\rho_{b} h_{b}+\rho_{l} h_{l}\right) / h$ in the ABH area are also implemented due to the added mass of the damping layer. The equivalent Young's modulus of the sandwich plate that have to be inserted in Eq. (2b) for the compatibility equation on the Airy stress function, is deduced from the real bending stiffness $D(x)$ and the total thickness as $E(x)=$ $12\left(1-v^{2}\right) D(x) / h(x)^{3}$.

The contact force between the plate and the barrier is modelled with a penalty approach and a regularization with an Hertzian-like contact law. More Precisely, let us assume that 
the $N_{c}$ contacts are located at the discrete points $\left(x_{c i}, y_{c i}\right), i=1, \ldots, N_{c}$. Let $g(x, y)$ be the function prescribing the vertical position of the contact points. Then, the relative distance of the contact point to the plate plane can be denoted as $\eta(x, y, t)=g(x, y)-w(x, y, t)$. The contact force model $f(x, y, t)$ used then simply follows the rule

$$
f(x, y, t)=K_{c}[\eta(x, y, t)]_{+}^{\alpha},
$$

with $[.]_{+}$the positive part of $\eta$, stating that when $\eta$ is negative then the contact force vanishes, while it follows the power-law when interpenetration occurs; and $K_{c}$ is a parameter indicating the stiffness of the contact. This contact force derives from a potential $\psi(x, y, t)$ which reads

$$
f(x, y, t)=\frac{\partial \psi}{\partial \eta}, \quad \psi(x, y, t)=\frac{K_{c}}{\alpha+1}[\eta]_{+}^{\alpha+1}
$$

The local equation of motion is finally completed with the boundary conditions. In our study a free-edge condition is assumed on all edges, and the reader is referred to [27, 33, 35] for the detailed expressions.

\subsection{Modal approach}

The displacement $w$ and the Airy stress function $F$ are expressed as:

$$
w(x, y, t)=\sum_{k=1}^{N_{\Phi}} \Phi_{k}(x, y) q_{k}(t), F(x, y, t)=\sum_{n=1}^{N_{\Psi}} \Psi_{n}(x, y) \beta_{n}(t)
$$

where $q_{k}(t)$ and $\beta_{n}(t)$ are the modal coordinates, $N_{\Phi}$ and $N_{\Psi}$ the numbers of modes kept in the modal truncation. It is emphasized that the modes should satisfy the orthogonality conditions. For the transverse modes and in-plane modes, they respectively write

$$
\int_{S} \rho(x) h(x) \Phi_{k}(x, y) \Phi_{P}(x, y) \mathrm{d} S=\delta_{k p}, \quad \int_{S} \Psi_{k}(x, y) \Psi_{P}(x, y) \mathrm{d} S=\delta_{k p} .
$$

where $S$ is the plate surface, and $\delta_{k p}$ is the Kronecker delta function. After a classical Galerkin projection, one finally arrives at the nonlinear modal equations for the plate as

$$
\begin{aligned}
& \beta_{p}=-\frac{1}{2 \zeta_{p}^{4}} \sum_{i=1}^{N_{\Phi}} \sum_{j=1}^{N_{\Phi}} H_{i, j}^{p} q_{i} q_{j}, \\
& \ddot{q}_{s}+2 \xi_{s} \omega_{s} \dot{q}_{s}+\omega_{s}^{2} q_{s}=p_{s}+f_{s}+\frac{1}{M_{s}} \sum_{k=1}^{N_{\Phi}} \sum_{n=1}^{N_{\Psi}} E_{k, n}^{s} q_{k} \beta_{n},
\end{aligned}
$$

where $M_{s}=\int_{s} \rho(x) h(x) \Phi_{s}(x, y)^{2} d S$ is the modal mass, $p_{s}=\int_{s} p \Phi_{s} d S$ and $f_{s}=\int_{s} f \Phi_{s} d S$ are respectively modal external force and modal contact force. $H_{i, j}^{p}$ and $E_{k, l}^{s}$ are the coupling coefficients between each modes, whose expression reads

$$
H_{i, j}^{p}=\int_{S} \Psi_{p} L\left(\Phi_{i}, \Phi_{j}\right) d S, \quad E_{k, l}^{s}=\int_{S} L\left(\Phi_{k}, \Psi_{l}\right) \Phi_{s} d S
$$


It should be noted that the coefficients $H_{m, n}^{l}$ and $E_{k, l}^{s}$ satisfy the symmetry condition $H_{i, j}^{k}=H_{j, i}^{k}$ and $H_{i, j}^{k}=E_{k, j}^{i}$, which is deduced from the triple self-adjointness property of the Monge-Ampère operator $\mathcal{L}$, see [33, 34].

Due to the thickness variation, the eigenvalue problems have no analytical solution and need to be adressed numerically in order to obtain the family of solutions $\left(\omega_{k}, \Phi_{k}(x, y)\right)$, $\left(\zeta_{n}, \Psi_{n}(x, y)\right)$, as well as the damping ratios $\xi_{k}$, respectively. These computations have already been explicited in [27], and appendix A summarizes the main steps for the sake of completeness. Once the numerical modes obtained, the coefficients $H_{i, j}^{p}$ and $E_{k, l}^{s}$ are computed and stored once the geometry is defined. The equations of motion can then be numerically integrated with a dedicated solver which is explained in the next section.

\subsection{Time integration scheme}

The time integration scheme used in this work relies on earlier investigations that derived energy-conserving methods, both for the geometrically nonlinear plate problem, see [27, 34] and for the contact dynamics with a penalty approach, see [29, 36]. The main properties of the schemes are briefly recalled.

First of all, the modal amplitude is discretised as $q_{s}(t) \rightarrow q_{s}^{n}$, with $t_{n}=n \Delta t, \Delta t$ the time step and $n$ the current index. The geometrically nonlinear terms arising through polynomial couplings in the equations of motion can be treated in such a way to produce an energyconserving scheme $[27,34]$, which reads:

$$
\begin{aligned}
& \delta_{t t} q_{s}^{n}+2 \xi_{s} \omega_{s} \delta_{t} \cdot q_{s}^{n}+\omega_{s}^{2} q_{s}^{n}=\frac{1}{M_{s}} \sum_{k=1}^{N_{\Phi}} \sum_{n=1}^{N_{\Psi}} E_{k, l}^{s} q_{k}^{n}\left[\mu_{t} \cdot \beta_{l}^{n}\right]+p_{s}^{n}+f_{s}^{n}, \\
& \mu_{t} \beta_{p}^{n}=-\frac{1}{2 \zeta_{p}^{4}} \sum_{i=1}^{N_{\Phi}} \sum_{j=1}^{N_{\Phi}} H_{i, j}^{p} q_{i}^{n} q_{j}^{n-1},
\end{aligned}
$$

where the discrete operators $\delta_{t t}, \delta_{t}, \mu_{t}$. and $\mu_{t-}$ are detailed in appendix B.

For the contact force, the implicit nature of its modal expression renders the problem more difficult and the solution can be derived by using the linear relationship between physical and modal space. Let $\mathbf{x}$ denote any point in the plate with coordinates $(x, y)$. Let also $\mathbf{x}_{c i}$ be the location for $i$ th contact point, and gather all the contact points as $\mathbf{X}=\left[\mathbf{x}_{c 1}, \mathbf{x}_{c 2}, \ldots, \mathbf{x}_{c N_{c}}\right]$. Let us introduce $\mathbf{S}$ the modal matrix that contains the values of the $N_{\Phi}$ modes at the contact points, such that $S_{i j}=\Phi_{j}\left(\mathbf{x}_{c i}\right), \forall(i, j) \in\left\{1, \ldots, N_{c}\right\} \times\left\{1, \ldots, N_{\Phi}\right\}$. Let $\mathbf{u}^{n}=u(\mathbf{X}, n \Delta t)$ be the displacement at all the contact points and $\mathbf{q}^{n}=\left[q_{1}^{n}, q_{2}^{n}, \cdots q_{N_{\Phi}}^{n}\right]$ be a vector containing all the modal amplitudes, the relationship between the physical and modal spaces can be simply described as $\mathbf{u}^{n}=\mathbf{S q}^{n}$. With this relationship and by multiplying $\mathbf{S}$ in each side, Eq. (10) can be transferred to the physical space in which the contact force $\mathbf{f}^{\mathbf{n}}=f(\mathbf{X}, n \Delta t)$ can then be computed via

$$
\mathbf{f}^{\mathbf{n}}=\frac{\psi\left(\boldsymbol{\eta}^{n+1}\right)-\psi\left(\boldsymbol{\eta}^{n-1}\right)}{\boldsymbol{\eta}^{n+1}-\boldsymbol{\eta}^{n-1}},
$$

this choice being related to the derivation of a conservative scheme, as shown in $[36,37]$. Note finally that the scheme is of order two and implicit; a Newton-Raphson procedure is 
needed to compute the update because of the nonlinear character of the regularized restoring force describing the contact.

\section{Linear analysis}

In this section, a typical ABH plate is first defined. Then the linear characteristics are investigated, showing interesting features for the global behaviour of eigenfrequencies. Finally the linear performance of the plate $\mathrm{ABH}$ is documented in order to quantify properly its performance before adding the nonlinearities.

\subsection{Case study}

The geometrical and material parameters for the selected ABH plate are listed in Tab. 1. They have been selected as corresponding to typical values that can be used in an experimental set-up.

\begin{tabular}{lll}
\hline Plate & ABH & Layer \\
\hline$L=100 \mathrm{~cm}$ & $l_{a d d}=0$ & $x_{1}=0$ \\
$b=20 \mathrm{~cm}$ & $l_{A B H}=6 \mathrm{~cm}$ & $x_{2}=6 \mathrm{~cm}$ \\
$h_{0}=3 \mathrm{~mm}$ & $h_{t}=30 \mu \mathrm{m}$ & $h_{l}=100 \mu \mathrm{m}$ \\
$E_{b}=70 \mathrm{Gpa}$ & & $E_{l}=7 \mathrm{Gpa}$ \\
$\rho_{b}=2700 \mathrm{~kg} \cdot \mathrm{m}^{3}$ & & $\rho_{l}=1000 \mathrm{~kg} \cdot \mathrm{m}^{3}$ \\
$\eta_{b}=0.2 \%$ & & $\eta_{l}=80 \%$ \\
$\nu=0.3$ & & \\
\hline
\end{tabular}

Tab. 1: Parameters selected for the ABH plate.

Referring to Tab.1, the first column defines a uniform plate with thickness $h_{0}$ and given material parameters selected close to steel. This case will be used as a reference plate for the forthcoming simulations. The two next columns indicates the typical values defining the $\mathrm{ABH}$ tapered profile, with a very small value for the terminal thickness $h_{t}$. In this table, $l_{\text {add }}$ is set to zero, representing a typical ABH implementation without added platform of constant thickness. The value $l_{\text {add }}$ will then be increased in the simulations and be specified when needed. The third column defines the parameter values defining the viscoelastic layer. A convergence results on the eigenfrequencies have shown that when the grid dimension is selected as $500 \times 200$, the calculation is converged up to the $300^{\text {th }}$ frequency (which gives a value around $8000 \mathrm{~Hz}$ for the uniform plate). Consequently these values have been selected in all the forthcoming simulations for the plate grid.

\subsection{Modal analysis of the ABH plate}

The eigenvalues of the $\mathrm{ABH}$ plate is first investigated, as it provides crucial information on its linear damping performance. Considering the ABH plate defined in Tab. 1 with $l_{a d d}=0$, Fig. 2 shows the spectrum of the eigenfrequencies $\omega_{k}^{*}$ in the complex plane. 

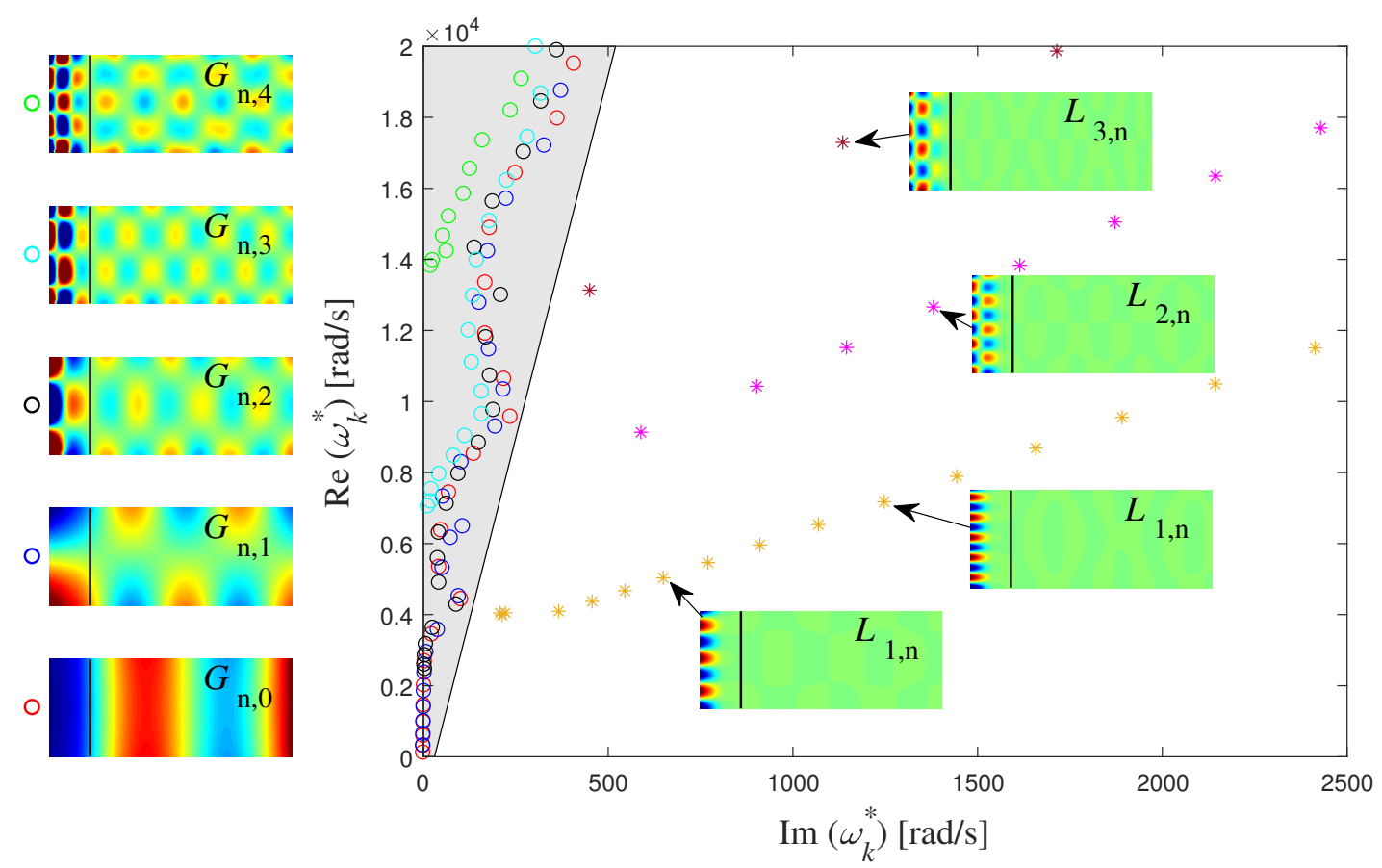

Fig. 2. Spectrum of eigenfrequencies $\omega_{k}^{*}$ in the complex plane for the ABH plate with parameters in Table 1. Global modes $G_{n, i}$, for $i=0, \ldots 4$, are represented by different colors of circles, and lies inside the gray region corresponding to small damping values. Each subset $G_{n, i}$ has the same number of nodal lines in the $y$ direction and one example of each family is shown on the left. Localized hyper-damped modes $L_{i, n}$ are depicted by different colors of stars. One example of each family is shown as inset to underline that they have the same number of half-waves in $x$ direction.

The striking feature is the very clear structure of organization of the eigenfrequencies in the complex plane, showing branches of solutions having the same trend. In order to give more insight, the modes have been classified following their belonging to each branch. First of all, stars have been used to depict hyper-damped modes, whose vibratory pattern show a strong localization in the tapered area, see [10]. In Fig. 2, the first three branches of solutions have been marked with stars of different colours and named $L_{1, n}, L_{2, n}$, and $L_{3, n}$. The first branch, $L_{1, n}$, marked with orange stars, shares for common point to have a single half-wave in the $x$-direction. When increasing $n$ along this branch, then the number of nodal lines in the $y$ direction is increasing, as reported in the inserted modal shapes in the figure, showing two different modes from this branch. The second branch $L_{2, n}$ is characterized by two half-waves in the $x$ direction and thus a nodal line in $x$ in the ABH region. Accordingly, the third branch $L_{3, n}$ reveals two nodal lines in the $x$ direction, and then, varying $n$, an increasing number of waves in $y$-direction. All these branches seem to have a constant behaviour at large amplitude close to linear, and tend to a value that could be defined as a cut-on frequency for each family. Interestingly, the cut-on frequency of the whole plate then corresponds to the first cut-on obtained for family $L_{1, n}$.

On the other hand, global modes are also numerous, and one can see that their damping 
capacity is small since they are all comprised in the grey-shaded area. Again, each of these mode shapes can be classified to make appear solution branches, marked with coloured circles in the figure. A typical example of a mode for each of these families that are denoted as $G_{n, 0}$, to $G_{n, 4}$, is shown on the left part of the figure. $G_{n, 0}$ with red circles corresponds to global modes having no nodal line along $y$ direction. Increasing $n$ along that family makes vary the number of half-waves in the $x$ direction. Following the same reasoning, $G_{n, 1}$ corresponds to the global modes having one nodal line at half the width $y=b / 2$, then $G_{n, 1}$ to modes having two nodal lines in $y$ direction, and so on.

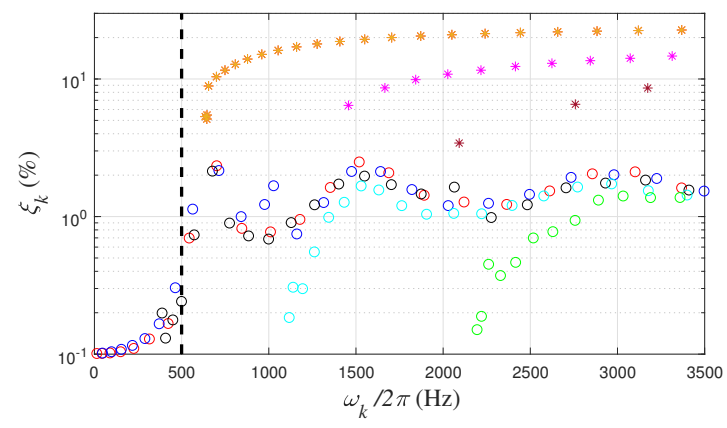

(a). $b=20 \mathrm{~cm}$

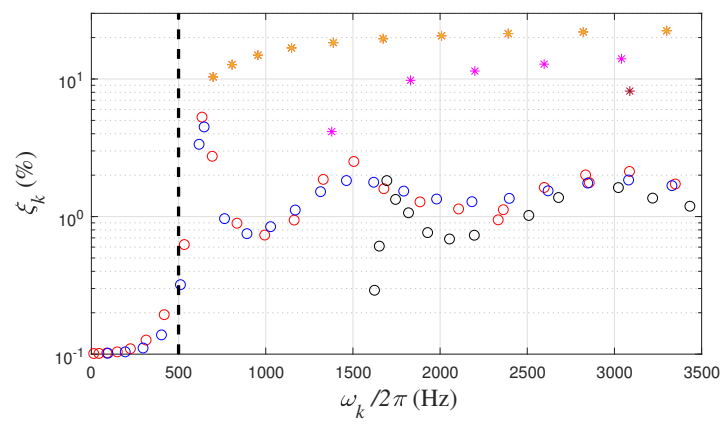

(b). $b=10 \mathrm{~cm}$

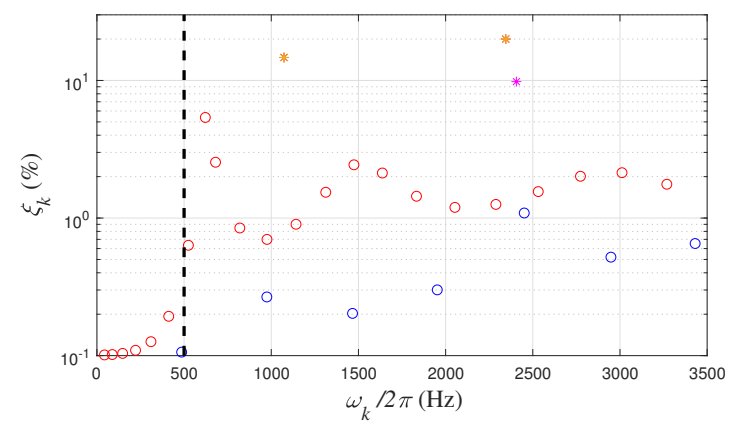

(c). $b=2 \mathrm{~cm}$

Fig. 3. Representation of the eigenvalues of the ABH plate (modal damping ratio versus eigenfrequencies) for three different values of the width $b$. (a) $b=20 \mathrm{~cm}$, (b) $b=10 \mathrm{~cm}$, (c) $b=2 \mathrm{~cm}$. The black dotted line at $500 \mathrm{~Hz}$ highlights the cut-on frequency at $500 \mathrm{~Hz}$ for each case.

Another representation of the same set of eigenfrequencies is shown in Fig. 3(a), where the modal damping ratios $\xi_{k}\left(\right.$ in $\%$ ) is represented as a function of the eigenfrequencies $\omega_{k} / 2 \pi$ (in $\mathrm{Hz}$ ). The structures of each branch of hyper-damped modes is again clearly evidenced. Taking $L_{1, n}$ (orange star) as an example, this type of modes have the highest damping ratios and increases as $n$ increases then tend to a threshold at around $22 \%$. One can also see that each branch of solution $L_{1, n}$ to $L_{3, n}$ starts at a given frequency that could be defined as the cut-on for each family. On the other hand, the global modes appear to saturate to a given small value of the modal damping ratio, between 1 and $2 \%$. A dashed black line defines the cut-on frequency for this $\mathrm{ABH}$ plate, at $500 \mathrm{~Hz}$. One can observe that below this 
cut-on, a set of underdamped mode remains, such that the efficacy of the ABH for vibration mitigation in the range $[0,500] \mathrm{Hz}$ will be poor.

Fig. 3(b-c) shows how the branch structure of the solutions for the plate case tends to disappear when the width $b$ is decreased so that one go from the plate to a beam-like structure. In Fig. 3(b), $b=10 \mathrm{~cm}$ while in Fig. $3(\mathrm{c}), b=2 \mathrm{~cm}$. The number of points corresponding to each branch of localized hyper-damped modes decreases rapidly and for $b=2 \mathrm{~cm}$, the branches are not visible anymore. One can also see the branches of solutions corresponding to global modes becoming more and more scarce. However a clear cut-on frequency stay constant for each case at $500 \mathrm{~Hz}$, only the number of modes below this cut-on decreases as $b$ does. Note that hyper-damped modes had already been noticed and studied in [10] for a beam. Here the analysis is extended to plates and deepened thanks to the specific $2 \mathrm{~d}$ area of the plate. Indeed all the localized modes from families $L_{i, n}$ with high damping ratios have a real $2 \mathrm{~d}$ pattern so that they are uniquely observed in this plate case with large $\mathrm{ABH}$ region, but almost disappears when one turns to beam-like ABH. We now turn to the characterization of the vibration response of the linear $\mathrm{ABH}$ plate.

\subsection{Linear performance of plate $A B H$}

The linear behaviour of the selected ABH plate is investigated thanks to the driving mobility in order to highlight its vibration mitigation capacity with a broadband excitation. Fig. 4 compares the point mobility at the excitation location $\mathbf{x}_{F}$ for the uniform plate (first column of Table 1), the ABH plate without added extension, and the $\mathrm{ABH}$ with an extension $l_{a d d}=4 \mathrm{~cm}$. A white noise excitation covering the frequency band $[0,3000] \mathrm{Hz}$, with amplitude $1 \mathrm{~N}$, is set at $x_{F}=0.7 \mathrm{~m}, y_{F}=0.14 \mathrm{~m}$, and the figure report the frequency dependence of the mobility up to $2000 \mathrm{~Hz}$.

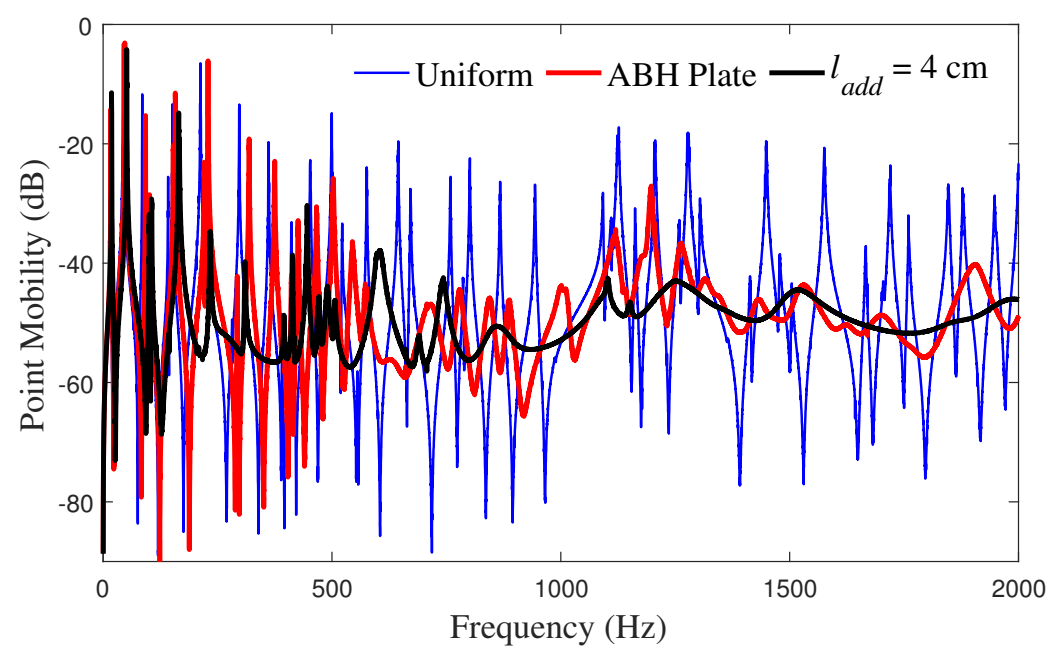

Fig. 4. Point mobilities of the uniform plate, the $\mathrm{ABH}$ plate and the $\mathrm{ABH}$ plate with $l_{\text {add }}=4 \mathrm{~cm}$ (parameters gathered in Table 1 . Structures are excited with a $[0,3000] \mathrm{Hz}$ white noise excitation, with amplitude $1 \mathrm{~N}$. Excitation and measurement point at $\left(x_{c}=0.7 \mathrm{~m}, \mathrm{y}_{\mathrm{c}}=0.14 \mathrm{~m}\right)$. 
Comparing the uniform plate with the $\mathrm{ABH}$, the usual impressive vibration mitigation is clearly retrieved in the the mobilities beyond the cut-on frequency (estimated in this case at $500 \mathrm{~Hz}$ ), with an important reduction of all the frequency peaks and a global smoothening effect of the frequency dependence. As already noted in [9] for beams, the effect of the added length $l_{\text {add }}$ is significant in the frequency band over the cut-on, with again an enhancement of the overall system damping in a broad frequency band.This effect can be attributed to the fact that the $\mathrm{ABH}$ region is lengthened, thus giving rise to a more important number of hyper-damped modes. As a consequence, the modal density and the modal overlap increases, leading to an improved global damping capacity. However the effect might not be so straightforward. Indeed, other simulation results computed with $l_{\text {add }}=2 \mathrm{~cm}$ and $l_{\text {add }}=6 \mathrm{~cm}$ showed that a fine tuning of all the parameters must be in order to find out the best added length, as also noticed in [9]. The optimal added length, found in our case for $l_{a d d}=4 \mathrm{~cm}$, is thus a subject for further studies, which is however not the purpose of the present paper.

Moving on the performance at the low frequency (i.e. below cut-on), one can observe that in the two $\mathrm{ABH}$ cases reported, a poor linear performance below the cut-on is always at hand. Although the peaks near the cut-on frequency have been reduced, for the very first frequencies, the resonance peaks remain sharp. Hence, if $l_{\text {add }}$ can to a certain extent reduces the value of the cut-on frequency -as a consequence of the enlargement of the total length of the ABH- it still does not lead to a decisive improvement for the first resonance peaks. In order to improve this low frequency performance, transfer of energy thanks to nonlinear mechanisms will now be studied. The coming sections aims at understanding the effect of vibro-impact and geometric nonlinearity to the system. For the latter case, the effect of $l_{\text {add }}$ will be further commented as it is a key factor in order to offer the possibility of triggering more easily the wave turbulence regime and thus to favour the energy transfer. We begin with the vibro-impact nonlinearity in the next section.

\section{Effect of the vibro-impact nonlinearity}

This section is devoted to the analysis of the vibro-impact nonlinearity alone, so that the geometrical nonlinearities are discarded from the model. Guided by the previous findings obtained for beams in [29], that demonstrate the efficiency of the energy transfer and gives clear guidelines for selecting the location of the contact points, the results will naturally extend to plate ABH. We begin by demonstrating the effect of a single contact point, then consider an optimal configuration with a small set of selected contacts. In this section, the additional length is not considered, $l_{a d d}=0$.

\subsection{Improvement of low-frequency efficiency with a single contact point}

In order to highlight how a single contact point can severely improve the efficacy, four different cases are compared. Case 1 and 2 corresponds to the uniform and ABH plate of the previous section. Case 3 is the uniform plate with a single contact point, selected so as to show how the contact nonlinearity effectively transfers rapidly the energy to the high frequency range. Finally case 4 is the $\mathrm{ABH}$ plate with the same single contact point. In 
the last two cases, the contact point is located at $\left(x_{c}=0.4 \mathrm{~m}, \mathrm{y}_{\mathrm{c}}=0.08 \mathrm{~m}\right)$. This location has been selected in order to avoid a point where too many nodal lines may cross. The optimal location will be investigated in the next section by showing how the arrangement of a few contact point can be very efficient. In any case the guidelines provided by the study on beams still holds [29], and one knows that contact points must be located at maxima of vibration (anti-nodes) of the eigenmodes whose frequency is below the cut-on. The numerical parameters for describing the contact law have been set as $\alpha=1.5, K_{c}=10^{11} \mathrm{~N} \cdot \mathrm{m}^{-1.5}, h_{c}=0$, in order to represent a grazing contact point with a stiff repelling force.

The plates are excited at a single input point, located at $\left(x_{F}=0.7 \mathrm{~m}, \mathrm{y}_{\mathrm{F}}=0.14 \mathrm{~m}\right)$ (also selected to avoid numerous nodal lines). A white noise excitation with amplitude $1 \mathrm{~N}$ is selected. In order to have a better picture of the energy transfer provided by the contact, two band noise excitations are considered: a full band $[0,3000] \mathrm{Hz}$, to investigate the global response of the plates, and a low frequency band $[0,500] \mathrm{Hz}$ in order to better ascertain the gain in the low-frequency range, below the cut-on frequency.

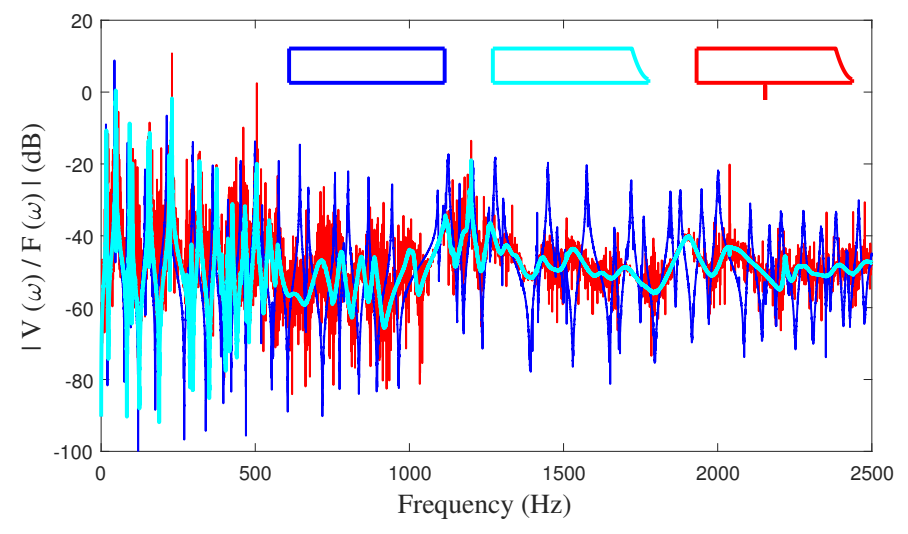

(a).

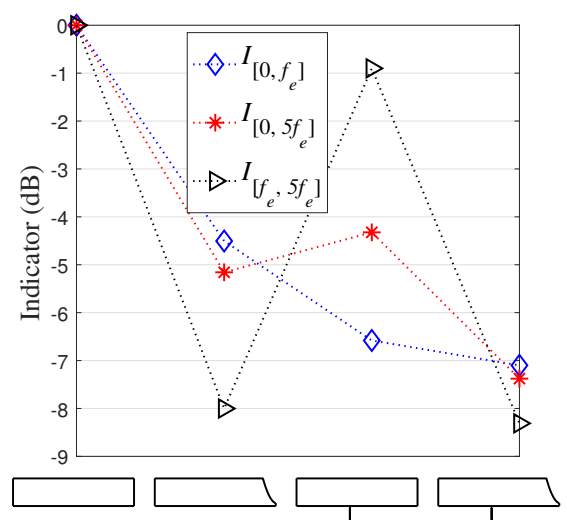

(b).

Fig. 5. (a) Frequency responses and (b) variations of indicators for cases 1 to 4 , for a full band $[0,3000] \mathrm{Hz}$ white noise excitation at $\left(x_{F}=0.7 \mathrm{~m}, \mathrm{y}_{\mathrm{F}}=0.14 \mathrm{~m}\right)$ with amplitude $1 \mathrm{~N},(\mathrm{a})$ : comparison of spectra $V(\omega) / F(\omega)$ calculated for case 1, case 2 , and case 4 , (b): $I_{\left[0,5 f_{e}\right]}, I_{\left[0, f_{e}\right]}$ and $I_{\left[f_{e}, 5 f_{e}\right]}$ calculated for all the four cases. Case 1: uniform plate. Case 2 : ABH plate. Case 3 : uniform plate with contact. Case 4 : VI-ABH plate.

We first report the response to the broadband $[0,3000] \mathrm{Hz}$ excitation in Fig. 5(a), where the normalized spectrum of $V(\omega) / F(\omega)$ (with $V(\omega)$ the velocity spectrum and $F(\omega)$ the forcing spectrum, both at excitation point) of cases 1,2 and 4 are represented. Only these three cases are represented in Fig. 5(a) for the sake of readability. The difference between the uniform and $\mathrm{ABH}$ plates have already been commented, the new feature is here the improvement brought by the contact point, which rapidly transfer energy to the high frequency range and tends to replace a smooth linear spectrum by a fuzzy curve with the important feature that the main resonant peaks located below the cut-on are eroded. This feature has already been addressed and commented in [29], and is not further investigated by dedicated zooms in this contribution, but the same conclusions applies as well. 
In order to give a more quantitative comparison, a frequency indicator comparing the energy of a given case to a reference one is introduced as

$$
I=10 \log _{10}\left(\frac{\int_{f_{0}}^{f_{\text {end }}} V_{c}^{2} \mathrm{~d} f}{\int_{f_{0}}^{f_{\text {end }}} V_{r e f}^{2} \mathrm{~d} f}\right),
$$

where $V$ is the output velocity spectrum at the excitation point $x=x_{F}$, and the subscript ref or $c$ stands respectively for the reference case and a current tested case. In particular, this indicator is used in Fig. 5(b) to ascertain the gains in different frequency bands: $I_{\left[0, f_{e}\right]}$, $I_{\left[0,5 f_{e}\right]}$, and $I_{\left[f_{e}, 5 f_{e}\right]}$ are calculated with $f_{e}=500 \mathrm{~Hz}$ (identified as the cut-on frequency) to evaluate respectively the low frequency, high frequency, and overall performance brought by a given test case. In this section, the reference case here is selected as the uniform plate without contact, and case 3 of the uniform plate with contact is also shown for better quantitative comparisons.

Fig. 5(b) shows the same feature as already observed for beams, with a strong improvement brought by the ABH termination when comparing case 1 and case 2, and again an interesting improvement when considering the VI-ABH, case 4 . The intermediate case 3 of the uniform plate with contact also shows that the strong energy transfer due to contact nonlinearity brings an improvement as compared to the uniform plate.

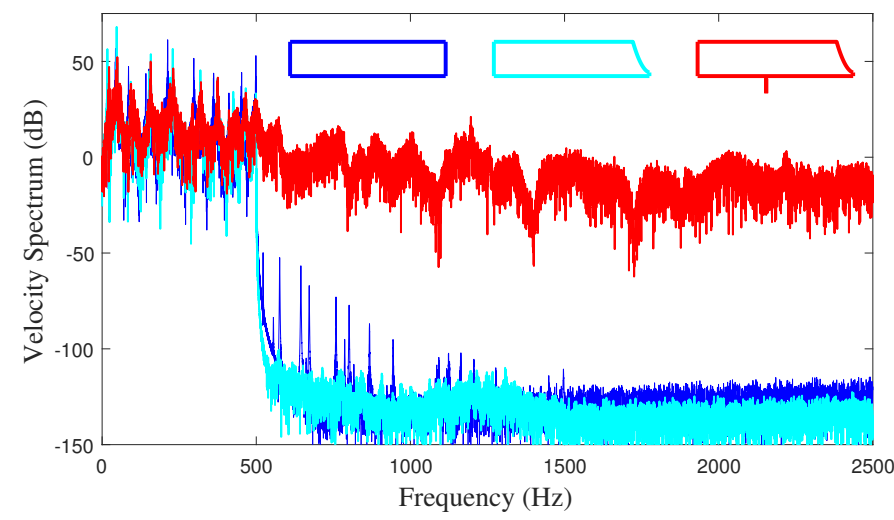

(a).

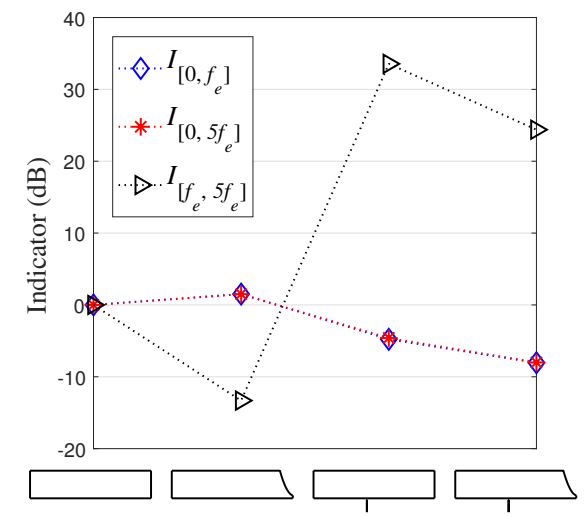

(b).

Fig. 6. (a) Frequency responses and (b) indicators to a low band $[0,500] \mathrm{Hz}$ white noise excitation at $\left(x_{F}=0.7 \mathrm{~m}, \mathrm{y}_{\mathrm{F}}=0.14 \mathrm{~m}\right)$ with amplitude $1 \mathrm{~N},(\mathrm{a})$ : velocity spectra calculated for case 1, case 2 , and case 4 , (b): $I_{\left[0,5 f_{e}\right]}, I_{\left[0, f_{e}\right]}$ and $I_{\left[f_{e}, 5 f_{e}\right]}$ calculated for all the four cases, Case 1: uniform plate. Case $2: \mathrm{ABH}$ plate. Case 3 : uniform plate with contact. Case $4:$ VI-ABH plate

Fig. 6 specifies the results to a noise excitation in the low-frequency range where the ABH effect is not effective. This is underlined by the slight increase of $I_{\left[0,5 f_{e}\right]}$ from case 1 to case 2. The nonlinear effect is clearly visible in Fig. 6(a) and the gain quantified by the decrease of the important indicators. Note that the behaviour of $I_{\left[f_{e}, 5 f_{e}\right]}$ is not meaningfull in this case since most of the energy is concentrated in the low frequency band, as attested by the fact that $I_{\left[0, f_{e}\right]}$ and $I_{\left[0,5 f_{e}\right]}$ are very close to each other. 
All the core conclusions related to an $\mathrm{ABH}$ beam are easily generalized to the $2 \mathrm{D}$ case of an $\mathrm{ABH}$ plate, whatever by checking the spectra or the indicators at different frequency bands. All of these results show a very strong consistence with the beam case, demonstrating that the core mechanism of energy transfer from low frequency to the high frequency, and the ABH effect of highly efficient vibration reduction at high frequency could be well observed, hence improving the average performance of the $\mathrm{ABH}$ plate to a great extent. The next section deals with an optimization of the contact points by considering a small number of them, with the perspective of an experimental set-up.

\subsection{Setup for numerous contact points}

In order to obtain the best arrangement of contact points, the key is to put one of these to local maxima of the eigenmodes below the cut-on frequency. Fig. 7(a) shows three of the typical eigenmode shapes present below the cut-on, having either no, one or two nodal lines along the $y$ direction. Mode 2 gives an example of a beam-like mode with almost no dependence on the $y$ axis. To control one mode from this family, one has just to select properly the $x_{c}$ coordinate. The second family of mode, e.g. mode 8 , is a plate mode with a nodal line at $y=b / 2$. For controlling this kind of mode, a good location of contact points is close to the boundaries in $y$. Finally, a third family are symmetric with the centreline (see e.g. mode 13) with two nodal lines along $y$. In contrary to the second family, one need to set the contact point close to the centreline for this family of modes.

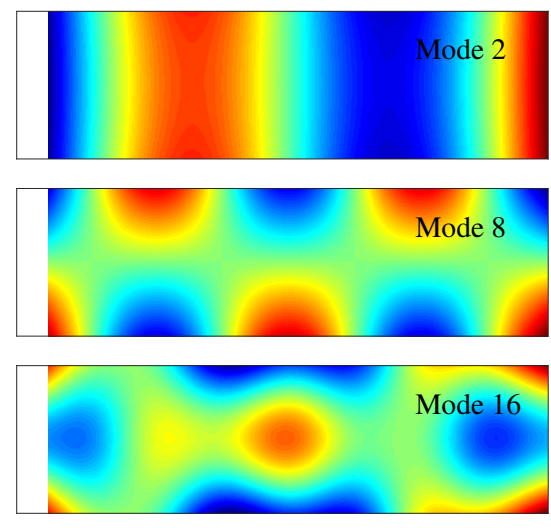

(a).
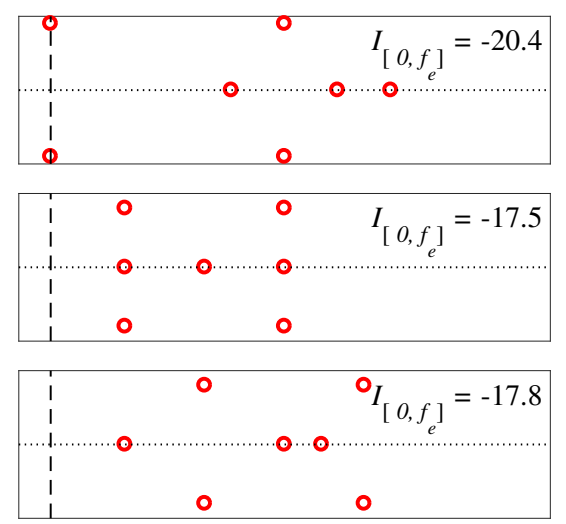

(b).

Fig. 7. (a) Three different types of modes below the cut-on frequency: simple beam-like mode belonging to $G_{n, 0}$ family (mode 2), torsional mode with nodal line at centre and belonging to $G_{n, 1}$ (mode 8), and symmetric plate mode about the centreline $y=0.1 \mathrm{~m}$ belonging to $G_{n, 2}$ (mode 16 ). (b): Three different arrangements of contact points and associated values of the gain in vibration mitigation given by $I_{\left[0, f_{e}\right]}$. The ABH region is located at the left of the dashed line.

Fig. 7(b) shows three different configurations of seven contact points, selected so as to fulfill the requirements of meeting the maximum number of anti-nodes of eigenmode shapes below the cut-on. It has been selected to show the results for 7 contact points since important 
gains as compared to a single point are found, without enforcing a too large number of points. The value of the indicator $I_{\left[0, f_{e}\right]}$ for each of these three cases, corresponding to a low-frequency excitation, is shown in the upper right part of each setup, showing that the gain in vibration mitigation can be very important for these three cases, with a maximum at $-20.4 \mathrm{~dB}$ for the first case. In this case, each contact point corresponds to the local maximum of one or several modes below the cut-on, which proves the fact that one should arrange the position of the contact points according to the mode shapes.

\section{Combined effect of contact and geometric nonlinearities}

The purpose of this section is to investigate the combined effect of contact and geometric nonlinearities on the performance of an $\mathrm{ABH}$ plate. As shown in [27], the wave turbulence regime with its associated energy flux through lengthscales allows transferring energy to improve the $\mathrm{ABH}$ performance. However the timescales for this regime to settle down are large, which is not the case for the contact nonlinearity. Hence combining the two effects should result in pairing their advantages. This is investigated in the next sections with a special emphasis first to the transient vibratory response of the plate to a shock, and the permanent regime in the case of forced excitation.

\subsection{Transient dynamics}

The transient response of different $\mathrm{ABH}$ plates to a shock excitation is simulated. The external excitation $p(x, y, t)$ is located in space at position $x_{F}=0.7 \mathrm{~m}, y_{F}=0.14 \mathrm{~m}$ and reads $p(x, t)=r(t) \delta\left(x-x_{F}\right) \delta\left(y-y_{F}\right)$ with $\delta$ the Dirac delta function and $r(t)$ the temporal content described by

$$
r(t)=\left\{\begin{array}{cl}
\frac{A}{2}\left[1+\cos \left(\frac{\pi\left(t-t_{0}\right)}{T_{w}}\right)\right] & \forall\left|t-t_{0}\right| \leq T_{w} \\
0 & \forall\left|t-t_{0}\right|>T_{w}
\end{array}\right.
$$

Fig. 8(a) illustrates the amplitude decay of the output displacement at the excitation point. The temporal parameters of the input force have been selected as $T_{w}=5 \mathrm{~ms}$, so that most of the energy sent to the plate is concentrated in the band $[0,200] \mathrm{Hz}$, below the cut-on. A strong amplitude of $A=200 \mathrm{~N}$ is considered to achieve large amplitude vibrations with strong excitation of the nonlinearities. In ordinate, the vibration amplitude $X=w\left(x_{F}, y_{F}, t\right)$ of the plate at the excitation point $\left(x_{F}, y_{F}\right)$ is shown. Reminding that the plate has free edges and a $3 \mathrm{~mm}$ of thickness in the uniform region, this means that the level of amplitude is very important, up to two times the thickness.

Five different plate cases are reported to understand the effect of each term in the vibration. First a linear ABH plate is considered, without the effect of neither contact nor geometric nonlinearity. Since the strike excitation is in the low-frequency band where the $\mathrm{ABH}$ is not effective, the vibration takes a long time to damp out. Adding only the geometric nonlinearity to consider the large amplitude vibrations occurring in the plate shows a slight decrease in the extinction time, meaning that even though large amplitudes motions are at hand, the wave turbulence only slightly transfers energy to high frequencies and marginally 
improve the damping capacity. Two cases including contact are then reported in black and magenta, with a single contact point located at $x_{c}=0.4 \mathrm{~m}$ and $y_{c}=0.08 \mathrm{~m}$, with stiffness $K_{c}=10^{11} \mathrm{~N} \cdot \mathrm{m}^{-1.5}$ as in the previous section. The black curve corresponds to a constant contact point at $h_{c}=0$, while in the other case, the contact point is removed after one second in order to see the changes brought by this kind of switch. The difference in the time response is noticeable but can be best appreciated in Fig. 8(b), where the energy decay in $E_{\sigma}$ at each time $t$ is calculated as

$$
E_{\sigma}(t)=\frac{\int_{t}^{T_{f}} X^{2} \mathrm{~d} \tau}{\int_{0}^{T_{f}} X^{2} \mathrm{~d} \tau}, \quad \forall t \in\left[0, T_{f}\right],
$$

where $T_{f}$ is the total time of the time response and $X$ the displacement of the beam.

These curves shows the drastic effect brough by the contact nonlinearity in order to damp out the vibration. Interestingly, when the contact is switched off after one second, the slope of energy decay is importantly changed and the performance becomes less effective. Since geometric nonlinearity is not considered in this case (black and magenta curves), one can observe that when turning the contact off then the final slope of energy decay is the same as in the first linear case, meaning that the only loss mechanisms at work are now the modal damping ratios of the plate.

Finally, when both geometric and contact nonlinearities are considered (cyan curve), their combined effect can bring a further improvement, representing the optimal performance among all the compared cases. The contact nonlinearity brings the most important effect, characterized by a very important decay in the very first milliseconds where numerous contacts are activated. The slope of the energy decay then decreases but taking into account the geometric nonlinearity gives a slight improvement.

A quantitative comparison can be drawn by calculating the slopes of the energy decay in $t \in[5,6] \mathrm{s}$. One obtains $-1.65 \mathrm{~dB} / \mathrm{s}$ for the linear case (blue), $-1.68 \mathrm{~dB} / \mathrm{s}$ when geometric nonlinearity (red) is considered, $-2.00 \mathrm{~dB} / \mathrm{s}$ for contact at the first second (magenta), $-2.99 \mathrm{~dB} / \mathrm{s}$ for the contact case (black), and $-3.00 \mathrm{~dB} / \mathrm{s}$ when both geometric nonlinearity and contact nonlinearity are considered (cyan). these trends confirm the observations that have found in Fig. 8(a). Both the geometric and contact nonlinearity can play positive roles in improving the performance of the $\mathrm{ABH}$ plate in the transient dynamics.

\subsection{Permanent regime}

The vibratory response in the permanent regime with a harmonic or noise excitation, is now investigated. This will allow for a better assessment of the improvement that can be awaited from the additional length $l_{a d d}$ in order to optimize the geometric nonlinearity and its associated energy flux.

Let us first illustrate the problem of time scale and the timeliness of the energy transfer, by considering an $\mathrm{ABH}$ plate with added platform of length $l_{\text {add }}=4 \mathrm{~cm}$ and without damping layer. A harmonic forcing with frequency $123 \mathrm{~Hz}$ is sent to the plate, with an increasing amplitude from 0 to $24 \mathrm{~N}$ in 8 seconds, in order to excite progressively the nonlinearities and observe the time scales related to the energy flux. Fig. 9 shows the velocity spectrograms 

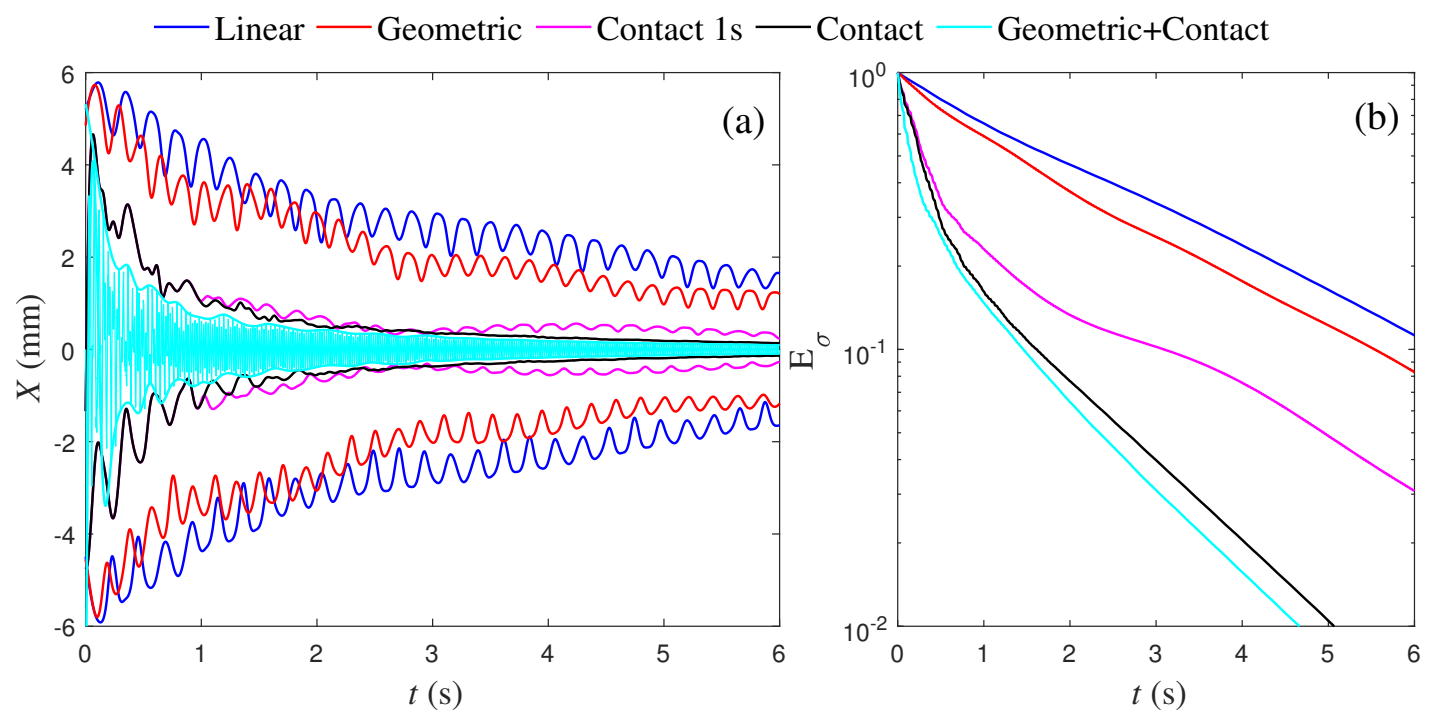

Fig. 8. Amplitude and Energy decay compared at different cases under a impulse excitation with time width $T_{w}=5 \mathrm{~ms}$ and amplitude $A=200 \mathrm{~N}$, (a): Envelope decay of the output displacement at the excitation point, (b): Energy decay curves.

of the plate for two different cases: (a) without contact point so that only the geometric nonlinearity is acting, (b) with contact point.

When only the geometric nonlinearity is considered, the spectrogram shows a transition from periodic regime to the wave turbulence, following the general features already unfolded for plate and shells, see e.g. [38, 39]. In particular, one can observe that the energy flux is active from around 4 seconds with a clear broadband spectrum, signature of the wave turbulence. When a single contact point is added, located at $x_{c}=40 \mathrm{~cm}, y_{c}=8 \mathrm{~cm}$, Fig. 9 (b) shows a completely different picture with appearance of the broadband spectrum for the first instants. This shows that both mechanisms will then be more efficiently and rapidly activated, without the large and needed timescales associated to wave turbulence, a quantification of which is given in [40].

We now come back to the case of band-limited noise excitation, where the forcing frequencies are limited to the range $[0,500] \mathrm{Hz}$, in order to investigate how the combination of nonlinearities can improve the low-frequency efficiency of the ABH. The amplitude of the forcing is $1 \mathrm{~N}$ and it is located at $x_{F}=0.4 \mathrm{~m}, y_{F}=0.08 \mathrm{~m}$.

Fig. 10 compares four different $\mathrm{ABH}$ plates by plotting the velocity spectrum of the response at the excitation point. The first case (dark blue) is the simple ABH plate without contact points nor added length $\left(l_{a d d}=0\right)$, so that the geometric nonlinearity is poorly excited. One can see that the plate behaves almost linearly with a negligible energy over $500 \mathrm{~Hz}$. The second case considers an added length of $l_{a d d}=4 \mathrm{~cm}$. Thanks to this extended platform, the geometric nonlinearity is more importantly excited, and it can be observed that a non-negligible fraction of the energy is now transmitted over the excitation band. The next case is with $l_{a d d}=0$ but addition of a single contact point located at $x_{c}=0.4 \mathrm{~m}, y_{c}=0.08 \mathrm{~m}$, 


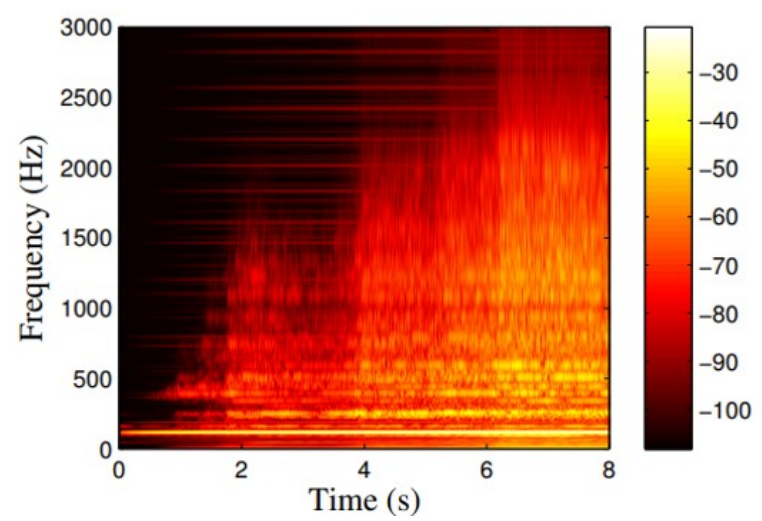

(a).

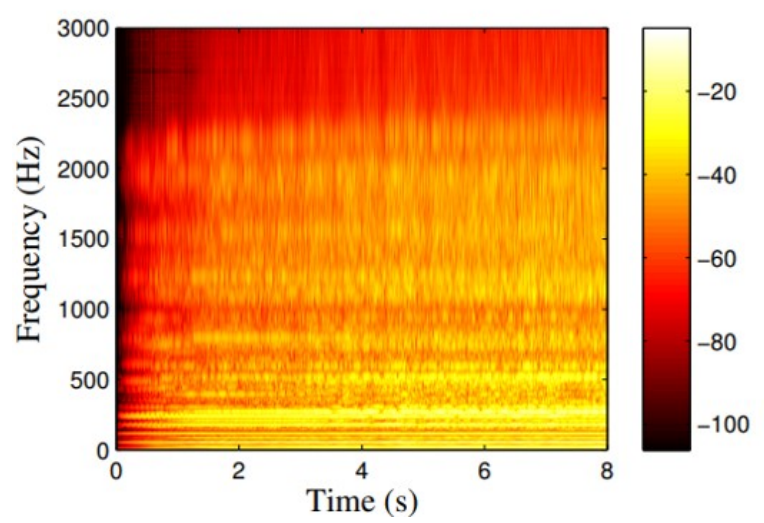

(b).

Fig. 9. Spectrograms of the displacement $X=w\left(x_{F}, y_{F}, t\right)$ for the ABH plate with additional length $l_{a d d}=4 \mathrm{~cm}$ and under a $123 \mathrm{~Hz}$ harmonic excitation (located at $x_{F}=70 \mathrm{~cm}, y_{F}=14 \mathrm{~cm}$ ), (a): without contact (b) with contact point (location $x_{c}=40 \mathrm{~cm}, y_{c}=8 \mathrm{~cm}$ ).

$l_{\text {add }}=0$, No contact $\_l_{\text {add }}=4 \mathrm{~cm}$, No contact $\_l_{\text {add }}=0$, Contact $\_l_{\text {add }}=4 \mathrm{~cm}$, Contact
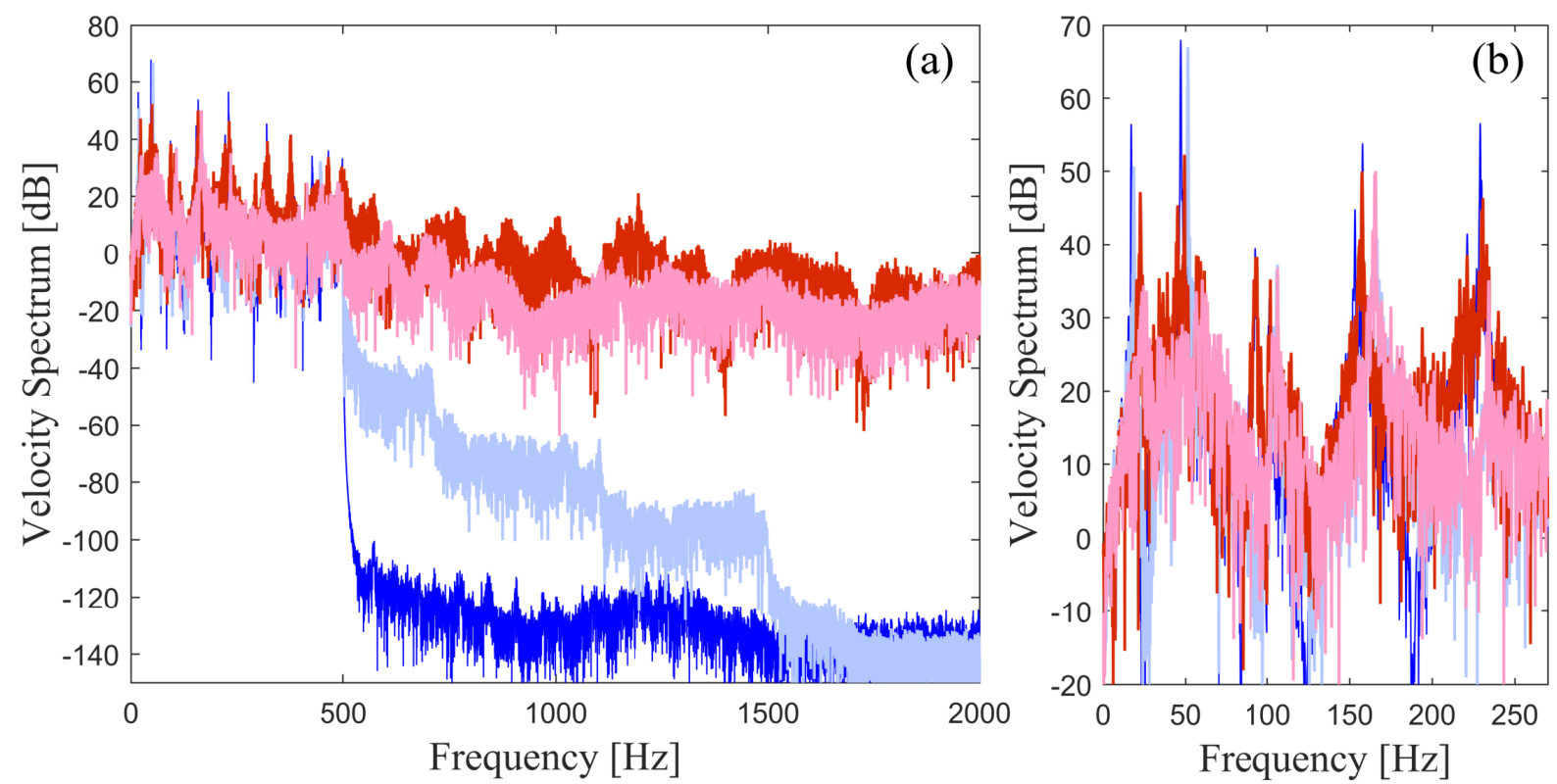

Fig. 10. Velocity spectrum for $\mathrm{ABH}$ plate of $l_{\text {add }}=0$ and $l_{\text {add }}=4 \mathrm{~cm}$ with and without contact, external excitation is a low frequency white noise in $[0,500] \mathrm{Hz}$ with amplitude $1 \mathrm{~N}$ and excited at $x_{F}=0.7 \mathrm{~m}, y_{F}=0.14 \mathrm{~m}$, contact cases are: single contact point located at $x_{c}=0.4 \mathrm{~m}$ and $y_{c}=0.08 \mathrm{~m}$, with contact stiffness $K_{c}=10^{11} \mathrm{~N} \cdot \mathrm{m}^{-1.5}$, (a): global view, (b): zoom in $[0,270] \mathrm{Hz}$ for the low frequency peaks.

with contact stiffness $K_{c}=10^{11} \mathrm{~N} \cdot \mathrm{m}^{-1.5}$. The energy transfer is now much more pronounced, which leads, as a consequence, to an appreciable decrease of the first peaks, below the cut-on. 
Finally the last case considers both effects, added length $l_{\text {add }}=4 \mathrm{~cm}$ and contact points. One can again observe a significant improvement in both frequency bands. Below the cut-on, the peaks decreases again in amplitude thanks to an improved energy transfer. Over the cut-on, a better damping capacity is also achieved thanks to the excellent linear properties of ABH plate with $l_{\text {add }}=4 \mathrm{~cm}$, already reported in the linear study, Fig. 4 .

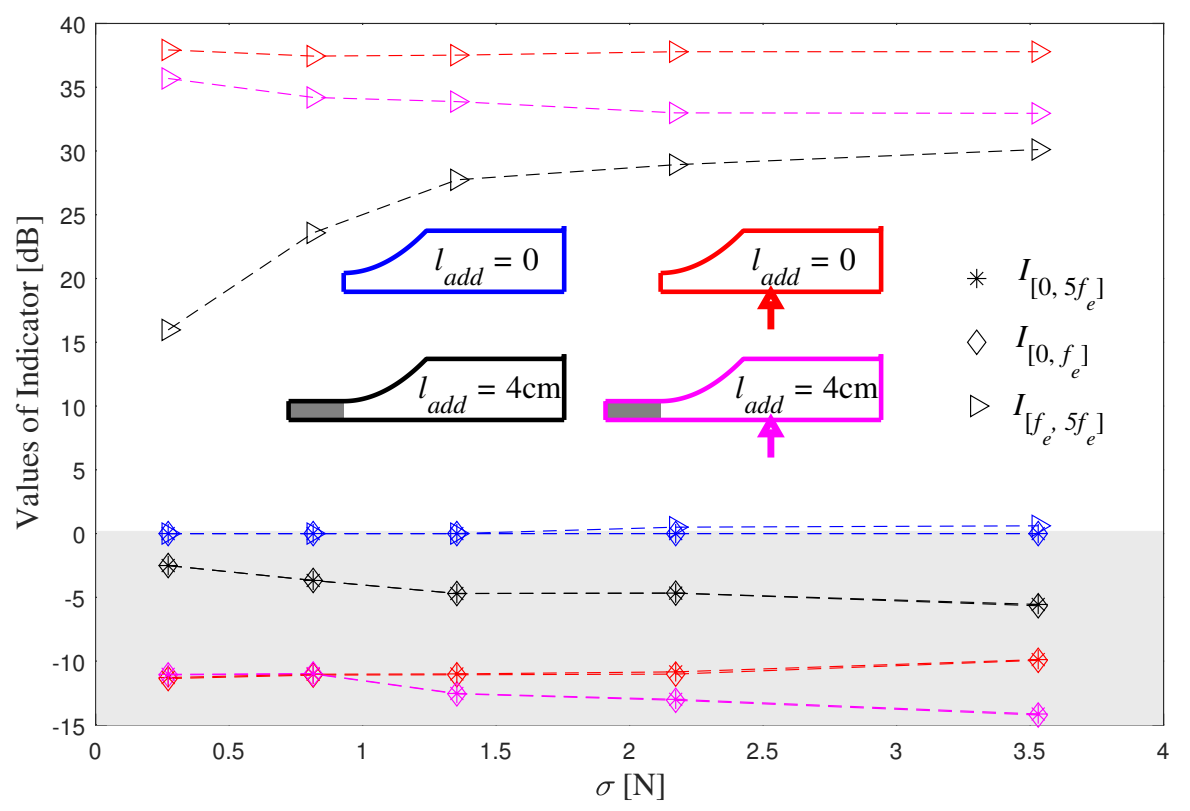

Fig. 11. Variation of indicators $I_{\left[0,5 f_{e}\right]}$ (star), $I_{\left[0, f_{e}\right]}$ (diamond), and $I_{\left[f_{e}, 5 f_{e}\right]}$ (triangle) under $[0,500] \mathrm{Hz}$ white noise as functions of the excitation level, compared among four cases: $l_{\text {add }}=0$ without contact, $l_{a d d}=0$ with contact, $l_{\text {add }}=4 \mathrm{~cm}$ without contact, and $l_{\text {add }}=4 \mathrm{~cm}$ with contact.

In order to obtain a more complete and quantitative picture, the values of the indicators $I_{\left[0, f_{e}\right]}, I_{\left[0,5 f_{e}\right]}$, and $I_{\left[f_{e}, 5 f_{e}\right]}$ for the above four cases are now displayed in Fig. 11. Also the amplitude of the forcing is varied for contrasting the results when the level of nonlinearity is increased. The standard variation of the white noise excitation $\sigma$ is thus varied from $0.25 \mathrm{~N}$ to $3.5 \mathrm{~N}$. The reference case for the calculation of the indicators is now selected as the non-contact ABH plate with $l_{\text {add }}=0$ under $\sigma=0.25 \mathrm{~N}$. And all the velocity spectra are normalized with the square variation of the excitation $\sigma^{2}$ before each calculation.

For the non-contact case with $l_{\text {add }}=0$, despite the increase of the excitation level, all the indicators remain almost constantly at 0 , although $I_{\left[f_{e}, 5 f_{e}\right]}$ shows small increase at $\sigma>2$, meaning that in this range of amplitude this reference case remains mostly linear. With $l_{\text {add }}=4 \mathrm{~cm}$ and without contact, the low frequency indicator $I_{\left[0, f_{e}\right]}$ decreases in average to $-5 \mathrm{~dB}$, while the high frequency indicator $I_{\left[f_{e}, 5 f_{e}\right]}$ increases about $15 \mathrm{~dB}$ to $30 \mathrm{~dB}$, highlighting the energy transfer. As in the cases reported in Fig. 6(b), most of the vibrational energy is concentrated within the low frequency range $\left[0, f_{e}\right]$, which means that the large increase $(15$ to $30 \mathrm{~dB})$ of $I_{\left[f_{e}, 5 f_{e}\right]}$ is negligible as compared to the total energy. This is ascertained in Fig. 11 where the full band indicator $I_{\left[0,5 f_{e}\right]}$ and the low frequency indicator $I_{\left[0, f_{e}\right]}$ are again 
almost coincident for all the cases considered. As expected, increasing the amplitude of the forcing creates stronger geometric nonlinearities, better energy flux, and improved vibration mitigation, a result that was already reported in [27].

Analyzing now the two cases with contact, one can onserve a global better performance in terms of $I_{\left[0, f_{e}\right]}$ for case 3 with $l_{a d d}=0$ and a single contact point, with a general gain in performance around 10dB. This gain in efficiency due to contact is found to be only slightly dependent on amplitude, meaning that the full transfer is already active even for very small values of vibration. Finally, for the case with $l_{\text {add }}=4 \mathrm{~cm}$ and contact, the coexistence of both nonlinearities shows that one can now retrieve a behaviour of $I_{\left[0, f_{e}\right]}$ that depends on vibration amplitude, so that reaching higher amplitudes gives rise to a better efficacy in terms of passive control.

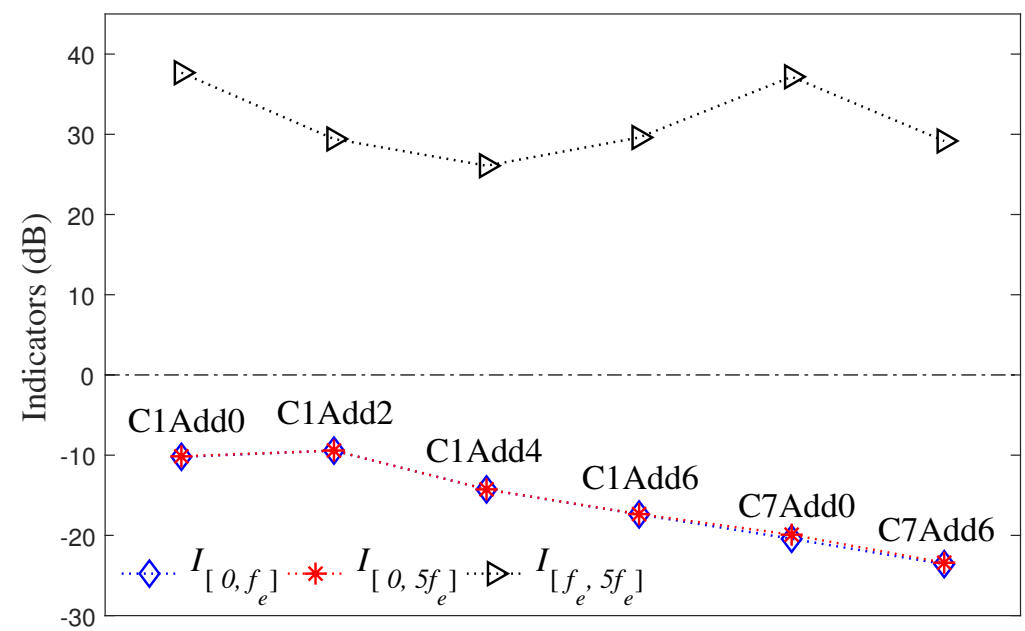

Fig. 12. variation of indicators as the small thickness extension $l_{\text {add }}$ varies, cases C1Add0-C1Add6: single contact point with extension $l_{\text {add }}=0, l_{\text {add }}=2 \mathrm{~cm}, l_{\text {add }}=4 \mathrm{~cm}$, and $l_{\text {add }}=6 \mathrm{~cm}$, case C7Add0 and C7Add6: 7 contact points and with $l_{\text {add }}=0$ and $l_{\text {add }}=6 \mathrm{~cm}$

To conclude this analysis, let us investigate the optimal gains that could be awaited by combining both nonlinearities, by playing on two different parameters: (i) the added length $l_{\text {add }}$ used to enhance the geometric nonlinearity, (ii) the number of contact points to improve the gains due to vibro-impact. Six cases are considered and compared, they are denoted as C1Add0 to C7add6, where the number after the letter $\mathrm{C}$ refers to the number of contact points, and the number after the letters add corresponds to the added length. The six cases are C1Add0, C1Add2, C1Add4, C1Add6, C7Add0 and C7Add6, hence covering four different added length from 0 to $6 \mathrm{~cm}$ and two cases with either one or seven contact points. The case with one contact point is the one previously studied, while the configuration selected for the seven contact points corresponds to the best arrangement reported in Fig. 7(b).

Fig. 12 shows the results for the indicators obtained for the six cases, where the plates are excited with the noise band limited to $[0,500] \mathrm{Hz}$ and amplitude of $1 \mathrm{~N}$. One can see that with a single contact point, increasing $l_{\text {add }}$ leads to a significant improvement, showing 
that the geometric nonlinearity can bring about a real gain. Going then to seven contact points without extension, the indicator continues to decrease showing that C7Add0 has a better global performance than C1Add6. Of course the best performance is obtained for C7Add6, with an impressive reduction of $24 \mathrm{~dB}$ as compared to the $\mathrm{ABH}$ plate case without any nonlinearity.

\section{Conclusion}

This paper investigates the vibration mitigation induced by the combined effects of geometric and impact nonlinearities introduced in an $\mathrm{ABH}$ plate. Taking advantage of the energy transfers from the low to the high frequency range, the global performance of the device is improved as compared to the case of a classical linear $\mathrm{ABH}$. The performance of the linear ABH damping effect, the vibro-impact dynamics, and the geometric nonlinearity are studied separately and in combination, in order to evaluate their contribution to the global mitigation effect.

Concerning the linear characteristics of the $\mathrm{ABH}$ plate, the numerical results report that varying the width of the plate has almost no effect on the value of the cut-on frequency of the ABH, but only modify the number of modes below it. Comparing to an ABH beam, it has been found that a special type of hyper-damped modes exist in the ABH plate, all of which being highly localized in the $\mathrm{ABH}$ area and gathered in branch of solutions having a very clear structure in the complex plane.

Considering independently the effect of contact nonlinearity, all the core conclusions drawn for a VI-ABH beam have been generalized to the $2 \mathrm{D}$ case of an $\mathrm{ABH}$ plate, and the gains in efficiency have been quantified properly. The combined effects of contact and geometric nonlinearities have been reported, showing that their respective advantages are advantageously added in order to enhance the overall efficacy. In particular, contact nonlinearity breaks the long timescales associated to energy flux due to geometric nonlinearity and favours immediate added efficacy.

The next steps of this research should consider experimental validations of these findings. Also, further investigations in order to analyse more deeply the branch structure of the $\mathrm{ABH}$ plate and the resulting cut-on frequencies could add more insights thanks to analytical developments on dispersion relationship.

\section{Appendix A Solving the eigenvalue problem}

The eigenvalue problem is solved using a finite difference method. Due to the variations of the ABH profile, an nonuniform grid is considered, according to [10, 27]: a coordinate change is introduced on $x$ in order to map the physical coordinate $x \in[0, L]$ onto a uniform mesh grid $\lambda \in[0,1]$. The one-to-one map between $\lambda$ and $x$ is selected according to the variations of the flexural wavelength and reads :

$$
\lambda(x)=\frac{1}{\bar{X}} \int_{0}^{x} \frac{1}{\sqrt{h(\theta)}} \mathrm{d} \theta, \text { with } \bar{X}=\int_{0}^{L} \frac{1}{\sqrt{h(\theta)}} \mathrm{d} \theta .
$$


and the second derivative with respect to $x$ can thus be written as

$$
\frac{\partial^{2} w}{\partial x^{2}} \approx \delta_{x x}=\frac{h_{l}^{-1 / 2}}{\bar{X}^{2}} \delta_{\lambda+}\left(\left(\mu_{\lambda-} h_{l}^{-1 / 2}\right) \delta_{\lambda-} w_{l, m}\right)
$$

while in the $y$ direction, the traditional uniform grid is still used. Finally the eigenvalued problems to be solved in the discrete manner is

$$
\left(\delta_{x x}+\delta_{y y}\right)\left(D_{l}\left(\delta_{x x}+\delta_{y y}\right) w_{l, m}\right)-(1-v)\left(\delta_{x x} D_{l}\right) \delta_{y y} w_{l, m}-\rho_{l} h_{l} \omega^{2} w_{l, m}=0
$$

As mentioned above, in the von Kármán equation for a plate, the eigenvalue problem includes the transverse displacement $w$ and the Airy stress function $F$. Regarding to the eigenvalue problem of $w$, Eq. (A.3) should be solved in two steps. In the first step, one use the real bending stiffness $D(x)$ and the purpose is to determine the eigenfrequencies and eigenmodes $\left(\omega_{k}, \Phi_{k}(x, y)\right)$; In the second step, the complex bending stiffness defined in Eq. (3) is considered. Complex eigenfrequencies $\omega_{k}^{*}$ are obtained, which permits to compute equivalent modal damping ratio $\xi_{k}$ from

$$
j \omega_{k}^{*}=\omega_{k}\left(-\xi_{k} \pm j \sqrt{1-\xi_{k}^{2}}\right),
$$

The eigenvalue problem for the Airy stress function $F$ is also solved from Eq. (A.3). In particular, for the movable boundary conditions, the eigenvalue problems for $F$ is equivalent to the eigenvalue problem of transverse displacement with clamped boundary condition. Hence, implying the clamped boundary condition in Eq. (A.3), one can solve the eigenvalue problem for $F$, which gives to the solution of $\left(\zeta_{n}, \Psi_{n}(x, y)\right)$. Once the numerical modes are obtained, the coefficients can $H_{i, j}^{p}$ and $E_{k, l}^{s}$ can thus be correspondingly obtained, and one finishes the solving procedure of all the eigenvalue problems.

\section{Appendix B Operators in Eq. (10)}

The operators appearing in Eq. $(10), \delta_{t t}, \delta_{t}$. are the difference operators which are defined respectively as

$$
\delta_{t t} q_{s}^{n}=\frac{q_{s}^{n+1}-2 q_{s}^{n}+q_{s}^{n-1}}{\Delta t^{2}}, \quad \delta_{t} \cdot q_{s}^{n}=\frac{q_{s}^{n+1}-q_{s}^{n-1}}{2 \Delta t},
$$

while $\mu_{t}$. and $\mu_{t-}$ are the averaging operators that read

$$
\mu_{t} . \beta_{l}^{n}=\frac{\beta_{l}^{n+1}+\beta_{l}^{n-1}}{2}, \quad \mu_{t-} \beta_{p}^{n}=\frac{\beta_{p}^{n}+\beta_{l}^{n-1}}{2} .
$$

\section{References}

[1] A. Pelat, F. Gautier, S.C. Conlon, and F. Semperlotti. The acoustic black hole: A review of theory and applications. Journal of Sound and Vibration, page 115316, 2020. 
[2] M.A. Mironov. Propagation of a flexural wave in a plate whose thickness decreases smoothly to zero in a finite interval. Soviet Physics Acoustics-USSR, 34(3):318-319, 1988.

[3] V.V. Krylov and F.J.B.S. Tilman. Acoustic 'black holes' for flexural waves as effective vibration dampers. Journal of Sound and Vibration, 274(3-5):605-619, 2004.

[4] V.B. Georgiev, J. Cuenca, F. Gautier, L. Simon, and V.V. Krylov. Damping of structural vibrations in beams and elliptical plates using the acoustic black hole effect. Journal of sound and vibration, 330(11):2497-2508, 2011.

[5] L. Tang, L. Cheng, H. Ji, and J. Qiu. Characterization of acoustic black hole effect using a one-dimensional fully-coupled and wavelet-decomposed semi-analytical model. Journal of Sound and Vibration, 374:172 - 184, 2016.

[6] K. Hook, J. Cheer, and S. Daley. A parametric study of an acoustic black hole on a beam. The Journal of the Acoustical Society of America, 145(6):3488-3498, 2019.

[7] D.J. O'Boy, V.V. Krylov, and V. Kralovic. Damping of flexural vibrations in rectangular plates using the acoustic black hole effect. Journal of Sound and Vibration, 329(22):4672-4688, 2010.

[8] M.R. Shepherd, C.A. McCormick, S.C. Conlon, and P.A. Feurtado. Modeling and optimization of acoustic black hole vibration absorbers. The Journal of the Acoustical Society of America, 141(5):4034-4034, 2017.

[9] L. Tang and L. Cheng. Enhanced acoustic black hole effect in beams with a modified thickness profile and extended platform. Journal of Sound and Vibration, 391:116 $126,2017$.

[10] V. Denis, A. Pelat, F. Gautier, and B. Elie. Modal overlap factor of a beam with an acoustic black hole termination. Journal of Sound and Vibration, 333(12):2475-2488, 2014.

[11] O. Aklouche, A. Pelat, S. Maugeais, and F. Gautier. Scattering of flexural waves by a pit of quadratic profile inserted in an infinite thin plate. Journal of Sound and Vibration, 375:38-52, 2016.

[12] J.Y. Lee and W. Jeon. Exact solution of Euler-Bernoulli equation for acoustic black holes via generalized hypergeometric differential equation. Journal of Sound and Vibration, 452:191-204, 2019.

[13] V.V. Krylov and R.E.T.B. Winward. Experimental investigation of the acoustic black hole effect for flexural waves in tapered plates. Journal of Sound and Vibration, 300(12):43-49, 2007. 
[14] V. Denis, F. Gautier, A. Pelat, and J. Poittevin. Measurement and modelling of the reflection coefficient of an acoustic black hole termination. Journal of Sound and Vibration, 349:67-79, 2015.

[15] E.P. Bowyer, D.J. O'Boy, V.V. Krylov, and F. Gautier. Experimental investigation of damping flexural vibrations in plates containing tapered indentations of power-law profile. Applied Acoustics, 74(4):553-560, 2013.

[16] P.A. Feurtado and S.C. Conlon. An experimental investigation of acoustic black hole dynamics at low, mid, and high frequencies. Journal of Vibration and Acoustics, 138(6):061002, 2016.

[17] H. Zhu and F. Semperlotti. Two-dimensional structure-embedded acoustic lenses based on periodic acoustic black holes. J. Appl. Physic, 122.6:065104, 2017.

[18] L. Zhao, S.C. Conlon, and F. Semperlotti. Broadband energy harvesting using acoustic black hole structural tailoring. Smart Materials and Structures, 23(6):065021, 2014.

[19] L. Lagny, M. Sécail-Géraud, J. Le Meur, S. Montrésor, K. Heggarty, C. Pezerat, and P. Picart. Visualization of travelling waves propagating in a plate equipped with $2 \mathrm{~d}$ abh using wide-field holographic vibrometry. Journal of Sound and Vibration, 461:114925, 2019 .

[20] J. Leng, V. Romero-García, A. Pelat, R. Picó, J-P. Groby, and F. Gautier. Interpretation of the acoustic black hole effect based on the concept of critical coupling. Journal of Sound and Vibration, page 115199, 2020.

[21] C.A. McCormick and M.R. Shepherd. Design optimization and performance comparison of three styles of one-dimensional acoustic black hole vibration absorbers. Journal of Sound and Vibration, 470:115164, 2020.

[22] C. Zhao and M.G. Prasad. Acoustic black holes in structural design for vibration and noise control. In Acoustics, volume 1, pages 220-251. Multidisciplinary Digital Publishing Institute, 2019.

[23] J. Deng, L. Zheng, P. Zeng, Y. Zuo, and O. Guasch. Passive constrained viscoelastic layers to improve the efficiency of truncated acoustic black holes in beams. Mechanical Systems and Signal Processing, 118:461 - 476, 2019.

[24] O. Aklouche, A. Wang, A. Pelat, and F. Gautier. Dispersion curves for bending waves in a meta-plate made with a periodic lattice of abh like scatterers. Proc. of Internoise Congress,Hong-Kong, 27-30 Aug. 2017.

[25] S.C. Conlon, J.B. Fahnline, and F. Semperlotti. Numerical analysis of the vibroacoustic properties of plates with embedded grids of acoustic black holes. The Journal of the Acoustical Society of America, 137(1):447-457, 2015. 
[26] V. Denis, A. Pelat, and F. Gautier. Scattering effects induced by imperfections on an acoustic black hole placed at a structural waveguide termination. Journal of Sound and Vibration, 362:56 - 71, 2016.

[27] V. Denis, A. Pelat, C. Touzé, and F. Gautier. Improvement of the acoustic black hole effect by using energy transfer due to geometric nonlinearity. International Journal of Non-Linear Mechanics, 94:134-145, 2017.

[28] T. Zhou, L. Tang, H. Ji, J. Qiu, and L. Cheng. Dynamic and static properties of double-layered compound acoustic black hole structures. International Journal of Applied Mechanics, 09(05):1750074, 2017.

[29] H. Li, C. Touzé, A. Pelat, F. Gautier, and X. Kong. A vibro-impact acoustic black hole for passive damping of flexural beam vibrations. Journal of Sound and Vibration, 450:28-46, 2019.

[30] H. Li, C. Touzé, A. Pelat, and F. Gautier. Combining nonlinear vibration absorbers and the acoustic black hole for passive broadband flexural vibration mitigation. International Journal of Non-Linear Mechanics, page 103558, 2020.

[31] V.E. Gusev, C. Ni, A. Lomonosov, and Z. Shen. Propagation of flexural waves in inhomogeneous plates exhibiting hysteretic nonlinearity: Nonlinear acoustic black holes. Ultrasonics, 61:126-135, 2015.

[32] A. Rekhy, R. Snyder, and J.M. Manimala. On the frequency up-conversion mechanism in metamaterials-inspired vibro-impact structures. Acoustics, (1):156-173, 2019.

[33] O. Thomas and S. Bilbao. Geometrically nonlinear flexural vibrations of plates: In-plane boundary conditions and some symmetry properties. Journal of Sound and Vibration, 315(3):569-590, 2008.

[34] M. Ducceschi and C. Touzé. Modal approach for nonlinear vibrations of damped impacted plates: Application to sound synthesis of gongs and cymbals. Journal of Sound and Vibration, 344:313-331, 2015.

[35] M. Ducceschi, C. Touzé, S. Bilbao, and C.J. Webb. Nonlinear dynamics of rectangular plates: investigation of modal interaction in free and forced vibrations. Acta Mechanica, 225:213-232, 2014.

[36] C. Issanchou, S. Bilbao, J.L. Le Carrou, C. Touzé, and O. Doaré. A modal-based approach to the nonlinear vibration of strings against a unilateral obstacle: Simulations and experiments in the pointwise case. Journal of Sound and Vibration, 393:229-251, 2017.

[37] S. Bilbao, A. Torin, and V. Chatziioannou. Numerical modeling of collisions in musical instruments. Acta Acustica united with Acustica, 101(1):155-173, 2015. 
[38] C. Touzé, S. Bilbao, and O. Cadot. Transition scenario to turbulence in thin vibrating plates. Journal of Sound and Vibration, 331(2):412-433, 2012.

[39] C. Touzé, O. Thomas, and M. Amabili. Transition to chaotic vibrations for harmonically forced perfect and imperfect circular plates. International Journal of Non-linear Mechanics, 46(1):234-246, 2011.

[40] M. Ducceschi, O. Cadot, C. Touzé, and S. Bilbao. Dynamics of the wave turbulence spectrum in vibrating plates: A numerical investigation using a conservative finite difference scheme. Physica D: Nonlinear Phenomena, 280:73-85, 2014. 